\title{
Observable heavy Higgs dark matter
}

\author{
Venus Keus, ${ }^{a, b}$ Stephen F. King, ${ }^{b}$ Stefano Moretti ${ }^{b, c}$ and Dorota Sokolowska ${ }^{d}$ \\ ${ }^{a}$ Department of Physics and Helsinki Institute of Physics, \\ Gustaf Hallstromin katu 2, FIN-00014 University of Helsinki, Finland \\ ${ }^{b}$ School of Physics and Astronomy, University of Southampton, \\ Southampton, SO17 1BJ, U.K. \\ ${ }^{c}$ Particle Physics Department, Rutherford Appleton Laboratory, \\ Chilton, Didcot, Oxon OX11 0QX, U.K. \\ ${ }^{d}$ University of Warsaw, Faculty of Physics, Pasteura 5, \\ 02-093 Warsaw, Poland \\ E-mail: Venus.Keus@helsinki.fi, King@soton.ac.uk, \\ S.Moretti@soton.ac.uk, Dorota.Sokolowska@fuw.edu.pl
}

ABstract: Dark Matter (DM), arising from an Inert Higgs Doublet, may either be light, below the $W$ mass, or heavy, above about $525 \mathrm{GeV}$. While the light region may soon be excluded, the heavy region is known to be very difficult to probe with either Direct Detection (DD) experiments or the Large Hadron Collider (LHC). We show that adding a second Inert Higgs Doublet helps to make the heavy DM region accessible to both DD and the LHC, by either increasing its couplings to the observed Higgs boson, or lowering its mass to $360 \mathrm{GeV} \lesssim m_{\mathrm{DM}}$, or both.

Keywords: Higgs Physics, Beyond Standard Model, Cosmology of Theories beyond the SM, Discrete and Finite Symmetries

ArXiv EPrint: 1507.08433 


\section{Contents}

1 Introduction 1

2 The I(2+1)HDM 3

2.1 The scalar potential 3

2.2 Mass eigenstates 4

2.3 Constraints on parameters 6

2.4 Simplified couplings in the $\mathrm{I}(2+1) \mathrm{HDM}$

3 Calculating the relic density in the I(2+1)HDM $\quad 7$

3.1 Relevant co-annihilation scenarios 8

3.2 The gauge limit 9

$\begin{array}{lll}3.3 & \text { The benchmark points } & 10\end{array}$

4 DM in the I(2+1)HDM: direct detection 12

4.1 Changes in $\Delta, \delta_{A}, \delta_{C}$

4.2 Changes in $m_{\mathrm{DM}} \quad 13$

$\begin{array}{lll}4.3 & \text { Direct detection } & 16\end{array}$

5 Heavy Higgs DM at the LHC in the I(2+1)HDM 17

$\begin{array}{lll}5.1 & \text { Monojet results } & 18\end{array}$

$\begin{array}{lll}5.2 & \text { Dijet VBF results } & 19\end{array}$

$\begin{array}{lll}5.3 \text { HS results } & 19\end{array}$

6 Conclusion $\quad 22$

A Feynman rules in the simplified I(2+1)HDM 23

$\begin{array}{ll}\text { B Feynman diagrams for relic density calculations } & 23\end{array}$

C Feynman diagrams for the LHC analysis $\quad 25$

C.1 Diagrams with monojet final states 25

C.2 VBF diagrams with dijet final states 26

C.3 HS diagrams with (on-shell) gauge boson final states 26

\section{Introduction}

The long-awaited Higgs boson, with a mass of $m_{h} \approx 125 \mathrm{GeV}$, was famously discovered in 2012 by the ATLAS and CMS experiments at the CERN Large Hadron Collider (LHC) $[1,2]$. Although its properties are in accordance with the predictions of the Standard Model (SM), including Electro-Weak (EW) precision data, it remains an intruiging 
possibility that the observed Higgs boson, denoted here as $h$, may just be one member of an extended Higgs sector. A good motivation for the latter is the idea that it might provide a candidate for Cold Dark Matter (CDM).

Although the nature of Dark Matter (DM) is not yet known, according to the Standard Cosmological Lambda-CDM Model [3] it should be a particle which is stable on cosmological time scales, cold, i.e., non-relativistic at the onset of galaxy formation, non-baryonic, neutral and weakly interacting. Various candidates for such a state exist in the literature, the most well-studied being the Weakly Interacting Massive Particles (WIMPs) [4-6], with masses between a few $\mathrm{GeV}$ and a few $\mathrm{TeV}$. Any such WIMP candidate must be cosmologically stable, usually due to the conservation of a discrete symmetry, and must freeze-out (i.e., drop out of thermal equilibrium) to yield the observed relic density [3]:1

$$
\Omega_{\mathrm{DM}} h^{2}=0.1199 \pm 0.0027
$$

It is clear that the SM Higgs sector cannot provide a WIMP candidate, since its Higgs boson is unstable. However, it was suggested some time ago that the Higgs sector could be extended by the addition of an extra doublet, which may not develop a Vacuum Expectation Value (VEV), leaving a discrete $Z_{2}$ symmetry unbroken [7]. Independently, it was later shown that an extra scalar doublet with zero VEV, odd under a discrete $Z_{2}$ symmetry, could yield monojets at hadron colliders while being constrained by DM considerations (the first time to our knowledge that any connection with hadron colliders or DM was made) [8]. This possibility, which became known as the Inert Doublet Model (IDM), has been studied extensively for the last few years (see, e.g., [9-11]). Since the IDM involves 1 Inert Doublet plus 1 active Higgs Doublet, we shall also refer to it henceforth as the $\mathrm{I}(1+1) \mathrm{HDM}$.

In the IDM, aka the I(1+1)HDM, one extra spin-zero $\mathrm{SU}(2)_{L}$ doublet with the same quantum numbers as the SM Higgs doublet is introduced. One of the possible vacuum states in this model involves the first doublet acquiring a VEV, henceforth called the active doublet, while the second doublet does not develop a VEV and is referred to as the inert doublet since it does not take part in EW Symmetry Breaking (EWSB). Since this doublet does not couple to fermions and it is by construction the only $Z_{2}$-odd field in the model, it provides a stable DM candidate, namely the lightest state among scalar and pseudo-scalar $Z_{2}$-odd particles.

The $\mathrm{I}(1+1)$ HDM remains a viable model for a scalar DM candidate, being in agreement with current experimental constraints. As of now, there are two regions of DM masses where one can expect viable solutions: a low DM mass region, $53 \mathrm{GeV} \lesssim m_{\mathrm{DM}} \lesssim m_{W}$ and a heavy DM mass region, $m_{\mathrm{DM}} \gtrsim 525 \mathrm{GeV}$. The most recent experimental data, both from direct detection experiments and from the LHC, has reduced the viable parameter space in the low mass region $[12,13]$. In the heavy mass region, however, where the sensitivity of DM direct detection experiments decreases significantly with increasing DM mass, the DM candidate may escape possible detection in this model.

\footnotetext{
${ }^{1}$ Since the Planck 2015 results quotes various results for $\Omega_{\mathrm{DM}} h^{2}$, depending on which spectra such as TT, TE and EE are used, we prefer to use here the Planck 2013 result whose error encompasses all of them.
} 
In a recent paper [14] we studied DM in a model with 2 inert Higgs plus 1 active Higgs doublet, which we referred to as the I $(2+1)$ HDM. In particular we focused on the region of parameter space of the $\mathrm{I}(2+1) \mathrm{HDM}$ where the DM candidate, the lightest inert scalar, is in the light mass region $\left(m_{\mathrm{DM}} \lesssim m_{W}\right)$. We found that the extended scalar sector can relax the exclusion limits from direct detection experiments, providing a viable DM candidate in a region of parameter space which would be excluded in the $\mathrm{I}(1+1)$ HDM. In this paper we study the heavy DM mass region of the I $(2+1)$ HDM. We show that heavy Higgs DM in this model becomes more readily observable as a result of either lowering the DM mass to $360 \mathrm{GeV} \lesssim m_{\mathrm{DM}}$, or increasing the DM-Higgs coupling, or both, while always maintaining the DM relic density within the required region.

The layout of the remainder of this paper is as follows. In section 2 we review the $\mathrm{I}(2+1) \mathrm{HDM}$ and focus on a simplified version of the model based on a smaller number of parameters. In section 3 we calculate the relic density in the $\mathrm{I}(2+1) \mathrm{HDM}$, discussing the relevant DM annihilation scenarios, including the extended co-annhilating (pseudo)scalar sector. Section 4 will be focused on new features of the $\mathrm{I}(2+1) \mathrm{HDM}$ with respect to the $\mathrm{I}(1+1) \mathrm{HDM}$ in the context of DM phenomenology, including enhanced DM-Higgs couplings and the new mass region $360 \mathrm{GeV} \lesssim m_{\mathrm{DM}} \lesssim 525 \mathrm{GeV}$ as well as on discussing the resulting improved prospects for direct detection. In section 5 we present heavy DM signals via Higgs mediation at the LHC in the $\mathrm{I}(2+1) \mathrm{HDM}$ which look more promising than in the $\mathrm{I}(1+1) \mathrm{HDM}$. Finally in section 6 we conclude the paper.

\section{The $\mathrm{I}(2+1) \mathrm{HDM}$}

\subsection{The scalar potential}

It has been shown in [15] that an N-Higgs-Doublet Model potential symmetric under a group $G$ of phase rotations can be divided into two parts; a phase invariant part, $V_{0}$, and a collection of extra terms ensuring the symmetry group $G, V_{G}$.

We construct our $Z_{2}$-symmetric 3 -Higgs Doublet Model potential generated by the group

$$
g=\operatorname{diag}(-1,-1,1)
$$

which is of the following form: ${ }^{2}$

$$
\begin{aligned}
V_{\mathrm{I}(2+1) \mathrm{HDM}}= & V_{0}+V_{Z_{2}} \\
V_{0}= & -\mu_{1}^{2}\left(\phi_{1}^{\dagger} \phi_{1}\right)-\mu_{2}^{2}\left(\phi_{2}^{\dagger} \phi_{2}\right)-\mu_{3}^{2}\left(\phi_{3}^{\dagger} \phi_{3}\right) \\
& +\lambda_{11}\left(\phi_{1}^{\dagger} \phi_{1}\right)^{2}+\lambda_{22}\left(\phi_{2}^{\dagger} \phi_{2}\right)^{2}+\lambda_{33}\left(\phi_{3}^{\dagger} \phi_{3}\right)^{2} \\
& +\lambda_{12}\left(\phi_{1}^{\dagger} \phi_{1}\right)\left(\phi_{2}^{\dagger} \phi_{2}\right)+\lambda_{23}\left(\phi_{2}^{\dagger} \phi_{2}\right)\left(\phi_{3}^{\dagger} \phi_{3}\right)+\lambda_{31}\left(\phi_{3}^{\dagger} \phi_{3}\right)\left(\phi_{1}^{\dagger} \phi_{1}\right) \\
& +\lambda_{12}^{\prime}\left(\phi_{1}^{\dagger} \phi_{2}\right)\left(\phi_{2}^{\dagger} \phi_{1}\right)+\lambda_{23}^{\prime}\left(\phi_{2}^{\dagger} \phi_{3}\right)\left(\phi_{3}^{\dagger} \phi_{2}\right)+\lambda_{31}^{\prime}\left(\phi_{3}^{\dagger} \phi_{1}\right)\left(\phi_{1}^{\dagger} \phi_{3}\right) . \\
V_{Z_{2}}= & -\mu_{12}^{2}\left(\phi_{1}^{\dagger} \phi_{2}\right)+\lambda_{1}\left(\phi_{1}^{\dagger} \phi_{2}\right)^{2}+\lambda_{2}\left(\phi_{2}^{\dagger} \phi_{3}\right)^{2}+\lambda_{3}\left(\phi_{3}^{\dagger} \phi_{1}\right)^{2}+\text { h.c. }
\end{aligned}
$$

\footnotetext{
${ }^{2}$ Note that adding extra $Z_{2}$-respecting terms such as $\left(\phi_{3}^{\dagger} \phi_{1}\right)\left(\phi_{2}^{\dagger} \phi_{3}\right),\left(\phi_{1}^{\dagger} \phi_{2}\right)\left(\phi_{3}^{\dagger} \phi_{3}\right),\left(\phi_{1}^{\dagger} \phi_{2}\right)\left(\phi_{1}^{\dagger} \phi_{1}\right)$ and/or $\left(\phi_{1}^{\dagger} \phi_{2}\right)\left(\phi_{2}^{\dagger} \phi_{2}\right)$ does not change the phenomenology of the model. The coefficients of these terms, therefore, have been set to zero for simplicity.
} 
We shall not consider $\mathrm{CP}$-violation in this paper, therefore, we require all parameters of the potential to be real.

The doublets are defined as

$$
\phi_{1}=\left(\begin{array}{c}
\phi_{1}^{+} \\
\frac{H_{1}^{0}+i A_{1}^{0}}{\sqrt{2}}
\end{array}\right), \quad \phi_{2}=\left(\begin{array}{c}
\phi_{2}^{+} \\
\frac{H_{2}^{0}+i A_{2}^{0}}{\sqrt{2}}
\end{array}\right), \quad \phi_{3}=\left(\begin{array}{c}
G^{+} \\
\frac{v+h+i G^{0}}{\sqrt{2}}
\end{array}\right),
$$

where $\phi_{1}$ and $\phi_{2}$ are the two inert doublets and $\phi_{3}$ is the one active doublet which plays the role of the SM Higgs doublet, with $h$ being the SM-Higgs boson and $G^{ \pm}, G^{0}$ are the would-be Goldstone bosons.

We assign $Z_{2}$ charges to each doublet according to the $Z_{2}$ generator in eq. (2.1): odd$Z_{2}$ charge to the inert doublets, $\phi_{1}$ and $\phi_{2}$, and even- $Z_{2}$ charge to the active doublet, $\phi_{3}$. It is clear that the symmetry of the potential is respected by the vacuum alignment $\left(0,0, \frac{v}{\sqrt{2}}\right)$. The neutral fields from the inert doublets could then in principle be DM candidates. These neutral fields are stabilised from decaying into SM particles as a result of the conserved $Z_{2}$ symmetry of the potential after EWSB.

To make sure that the entire Lagrangian and not only the scalar potential is $Z_{2}$ symmetric, we assign an even $Z_{2}$ parity to all SM particles, identical to the $Z_{2}$ parity of the only doublet that couples to them, i.e., the active doublet $\phi_{3}$. With this parity assignment Flavour Changing Neutral Currents (FCNCs) are avoided as the extra doublets are forbidden to couple to fermions by $Z_{2}$ conservation.

The Yukawa Lagrangian of the model is identical to the SM Yukawa Lagrangian, with $\phi_{3}$ playing the role of the SM Higgs doublet:

$$
\begin{aligned}
\mathcal{L}_{\text {Yukawa }}= & \Gamma_{m n}^{u} \bar{q}_{m, L} \tilde{\phi}_{3} u_{n, R}+\Gamma_{m n}^{d} \bar{q}_{m, L} \phi_{3} d_{n, R} \\
& +\Gamma_{m n}^{e} \bar{l}_{m, L} \phi_{3} e_{n, R}+\Gamma_{m n}^{\nu} \bar{l}_{m, L} \tilde{\phi}_{3} \nu_{n, R}+\text { h.c. }
\end{aligned}
$$

\subsection{Mass eigenstates}

The minimum of the potential sits at the point $\left(0,0, \frac{v}{\sqrt{2}}\right)$ with $v^{2}=\frac{\mu_{3}^{2}}{\lambda_{33}}$.

The mass spectrum of the scalar particles are as follows.

\section{- The fields from the active doublet}

The fields from the third doublet, $G^{0}, G^{ \pm}, h$, which play the role of the SM Higgs doublet fields have squared masses:

$$
\begin{aligned}
m_{G^{0}}^{2} & =m_{G^{ \pm}}^{2}=0 \\
m_{h}^{2} & =2 \mu_{3}^{2}
\end{aligned}
$$

\section{- The CP-even neutral inert fields}

The pair of inert neutral scalar gauge eigenstates, $H_{1}^{0}, H_{2}^{0}$, which are rotated by

$$
R_{\theta_{h}}=\left(\begin{array}{cc}
\cos \theta_{h} & \sin \theta_{h} \\
-\sin \theta_{h} & \cos \theta_{h}
\end{array}\right), \quad \text { with } \quad \tan 2 \theta_{h}=\frac{2 \mu_{12}^{2}}{\mu_{1}^{2}-\Lambda_{\phi_{1}}-\mu_{2}^{2}+\Lambda_{\phi_{2}}}
$$


into the mass eigenstates, $H_{1}, H_{2}$, have squared masses:

$$
\begin{aligned}
m_{H_{1}}^{2} & =\left(-\mu_{1}^{2}+\Lambda_{\phi_{1}}\right) \cos ^{2} \theta_{h}+\left(-\mu_{2}^{2}+\Lambda_{\phi_{2}}\right) \sin ^{2} \theta_{h}-2 \mu_{12}^{2} \sin \theta_{h} \cos \theta_{h} \\
m_{H_{2}}^{2} & =\left(-\mu_{1}^{2}+\Lambda_{\phi_{1}}\right) \sin ^{2} \theta_{h}+\left(-\mu_{2}^{2}+\Lambda_{\phi_{2}}\right) \cos ^{2} \theta_{h}+2 \mu_{12}^{2} \sin \theta_{h} \cos \theta_{h} \\
\text { where } \quad \Lambda_{\phi_{1}} & =\frac{1}{2}\left(\lambda_{31}+\lambda_{31}^{\prime}+2 \lambda_{3}\right) v^{2}, \quad \Lambda_{\phi_{2}}=\frac{1}{2}\left(\lambda_{23}+\lambda_{23}^{\prime}+2 \lambda_{2}\right) v^{2}
\end{aligned}
$$

\section{- The charged inert fields}

The pair of inert charged gauge eigenstates, $\phi_{1}^{ \pm}, \phi_{2}^{ \pm}$, which are rotated by

$$
R_{\theta_{c}}=\left(\begin{array}{cc}
\cos \theta_{c} & \sin \theta_{c} \\
-\sin \theta_{c} & \cos \theta_{c}
\end{array}\right), \quad \text { with } \quad \tan 2 \theta_{c}=\frac{2 \mu_{12}^{2}}{\mu_{1}^{2}-\Lambda_{\phi_{1}}^{\prime}-\mu_{2}^{2}+\Lambda_{\phi_{2}}^{\prime}}
$$

into the mass eigenstates, $H_{1}^{ \pm}, H_{2}^{ \pm}$, have squared masses:

$$
\begin{aligned}
m_{H_{1}^{ \pm}}^{2} & =\left(-\mu_{1}^{2}+\Lambda_{\phi_{1}}^{\prime}\right) \cos ^{2} \theta_{c}+\left(-\mu_{2}^{2}+\Lambda_{\phi_{2}}^{\prime}\right) \sin ^{2} \theta_{c}-2 \mu_{12}^{2} \sin \theta_{c} \cos \theta_{c} \\
m_{H_{2}^{ \pm}}^{2} & =\left(-\mu_{1}^{2}+\Lambda_{\phi_{1}}^{\prime}\right) \sin ^{2} \theta_{c}+\left(-\mu_{2}^{2}+\Lambda_{\phi_{2}}^{\prime}\right) \cos ^{2} \theta_{c}+2 \mu_{12}^{2} \sin \theta_{c} \cos \theta_{c} \\
\text { where } \quad \Lambda_{\phi_{1}}^{\prime} & =\frac{1}{2}\left(\lambda_{31}\right) v^{2}, \quad \Lambda_{\phi_{2}}^{\prime}=\frac{1}{2}\left(\lambda_{23}\right) v^{2}
\end{aligned}
$$

\section{- The CP-odd neutral inert fields}

The pair of inert pseudo-scalar gauge eigenstates, $A_{1}^{0}, A_{2}^{0}$, which are rotated by

$$
R_{\theta_{a}}=\left(\begin{array}{cc}
\cos \theta_{a} & \sin \theta_{a} \\
-\sin \theta_{a} & \cos \theta_{a}
\end{array}\right), \quad \text { with } \quad \tan 2 \theta_{a}=\frac{2 \mu_{12}^{2}}{\mu_{1}^{2}-\Lambda_{\phi_{1}}^{\prime \prime}-\mu_{2}^{2}+\Lambda_{\phi_{2}}^{\prime \prime}}
$$

into the mass eigenstates, $A_{1}, A_{2}$, have squared masses:

$$
\begin{aligned}
m_{A_{1}}^{2} & =\left(-\mu_{1}^{2}+\Lambda_{\phi_{1}}^{\prime \prime}\right) \cos ^{2} \theta_{a}+\left(-\mu_{2}^{2}+\Lambda_{\phi_{2}}^{\prime \prime}\right) \sin ^{2} \theta_{a}-2 \mu_{12}^{2} \sin \theta_{a} \cos \theta_{a} \\
m_{A_{2}}^{2} & =\left(-\mu_{1}^{2}+\Lambda_{\phi_{1}}^{\prime \prime}\right) \sin ^{2} \theta_{a}+\left(-\mu_{2}^{2}+\Lambda_{\phi_{2}}^{\prime \prime}\right) \cos ^{2} \theta_{a}+2 \mu_{12}^{2} \sin \theta_{a} \cos \theta_{a} \\
\text { where } \quad \Lambda_{\phi_{1}}^{\prime \prime} & =\frac{1}{2}\left(\lambda_{31}+\lambda_{31}^{\prime}-2 \lambda_{3}\right) v^{2}, \quad \Lambda_{\phi_{2}}^{\prime \prime}=\frac{1}{2}\left(\lambda_{23}+\lambda_{23}^{\prime}-2 \lambda_{2}\right) v^{2}
\end{aligned}
$$

We will refer to $\left(H_{1}, A_{1}, H_{1}^{ \pm}\right)$as the fields from the first generation and to $\left(H_{2}, A_{2}, H_{2}^{ \pm}\right)$ as the fields from the second generation. Each of the four neutral particles could, in principle, be the DM candidate, provided it is lighter than the other neutral states. In what follows, without loss of generality, we assume the CP-even ${ }^{3}$ neutral particle $H_{1}$ from the first generation to be lighter than all other inert particles, that is:

$$
m_{H_{1}}<m_{H_{2}}, m_{A_{1,2}}, m_{H_{1,2}^{ \pm}}
$$

(Note that this choice is arbitrary: if the CP-even particle from the second generation, $\mathrm{H}_{2}$, where to be assumed lighter than the other inert states, then $\mathrm{H}_{2}$ will play the role of the DM candidate.)

\footnotetext{
${ }^{3}$ For the CP-even particle to be the DM candidate rather than the CP-odd particle, it is required that $m_{H_{1}}<m_{A_{1}}$, which leads to $\lambda_{2}, \lambda_{3}<0$. If instead $A_{1}$ is assumed to be the DM candidate, $\lambda_{2}, \lambda_{3}>0$. Hence, the results of our analysis are also applicable to the $A_{1}$ DM case by changing the sign of $\lambda_{2}$ and $\lambda_{3}$.
} 
Assuming the CP-even neutral inert particles are lighter than the CP-odd and charged inert particles puts the following constraints on the parameters:

$$
2 \lambda_{2}, 2 \lambda_{3}<\lambda_{23}^{\prime}, \lambda_{31}^{\prime}<0 .
$$

In our DM analysis, we consider cases where the mass alignment is changed, but where $H_{1}$ is always the lightest inert state and hence is the DM particle. In the remainder of the paper the notations $H_{1}$ and DM particle will be used interchangeably.

\subsection{Constraints on parameters}

In [14], we have studied in detail the theoretical constraints; positivity of the mass eigenstates, bounded-ness of the potential, positive-definite-ness of the Hessian, and the LEP limits on the parameters of the potential. These limits have been taken into account in the present paper.

Our parameter choice is also compliant with all experimental constraints studied in [16] for the I(1+1)HDM, namely the Electroweak Precision Test (EWPT) bounds. In the $\mathrm{I}(2+1) \mathrm{HDM}$ studied here, we are considering heavy inert particles (with the lightest being $m_{H_{1}}>425 \mathrm{GeV}$ ) with large mass splittings (with $\delta_{a}=\delta_{c}=\Delta=1 \mathrm{GeV}$ in case $\mathrm{G}$ and $\delta_{a}=\delta_{c}=1 \mathrm{GeV}, \Delta=100 \mathrm{GeV}$ in case $\mathrm{H}$ ), which clearly satisfy the EWPT bounds presented in figure 1 in [16].

\subsection{Simplified couplings in the $\mathrm{I}(2+1) \mathrm{HDM}$}

Due to the large number of free parameters in the I $(2+1)$ HDM which makes it impractical to analyse the model in the general case, we focus on a simplified case where parameters related to the first inert doublet are $k$ times the parameters related to the second doublet

$$
\mu_{1}^{2}=k \mu_{2}^{2}, \quad \lambda_{3}=k \lambda_{2}, \quad \lambda_{31}=k \lambda_{23}, \quad \lambda_{31}^{\prime}=k \lambda_{23}^{\prime},
$$

resulting in

$$
\Lambda_{\phi_{1}}=k \Lambda_{\phi_{2}}, \quad \Lambda_{\phi_{1}}^{\prime}=k \Lambda_{\phi_{2}}^{\prime}, \quad \Lambda_{\phi_{1}}^{\prime \prime}=k \Lambda_{\phi_{2}}^{\prime \prime}
$$

without introducing any new symmetry to the potential. The motivation for this simplified scenario is that in the $k=0$ limit the model reduces to the well-known $\mathrm{I}(1+1) \mathrm{HDM}$. We assume no specific relation among the other parameters of the potential. It is important to note that the remaining quartic parameters do not influencethe discussed DM and LHC phenomenology of the model and thus their values have been fixed in agreement with the constraints discussed in section 2.3 and compliant with the results on unitarity obtained in [17].

The $\boldsymbol{k}=\mathbf{1}$ case. $\quad$ In this paper we focus on the $k=1$ case in the heavy DM mass region. ${ }^{4}$ The mass spectrum in this case is simplified to:

$$
\begin{array}{ll}
m_{H_{1}}^{2}=-\mu_{2}^{2}+\Lambda_{\phi_{2}}-\mu_{12}^{2}, & m_{H_{2}}^{2}=m_{H_{1}}^{2}+2 \mu_{12}^{2}, \\
m_{H_{1}^{ \pm}}^{2}=-\mu_{2}^{2}+\Lambda_{\phi_{2}}^{\prime}-\mu_{12}^{2}, & m_{H_{2}^{ \pm}}^{2}=m_{H_{1}^{ \pm}}^{2}+2 \mu_{12}^{2}, \\
m_{A_{1}}^{2}=-\mu_{2}^{2}+\Lambda_{\phi_{2}}^{\prime \prime}-\mu_{12}^{2}, & m_{A_{2}}^{2}=m_{A_{1}}^{2}+2 \mu_{12}^{2} .
\end{array}
$$

\footnotetext{
${ }^{4}$ Other scenarios with $k \neq 1$ are studied in [14].
} 
The quartic couplings in the potential can be written in terms of the masses of the physical particles as:

$$
\begin{aligned}
\lambda_{23}^{\prime} & =\frac{1}{v^{2}}\left(m_{H_{1}}^{2}+m_{A_{1}}^{2}-2 m_{H_{1}^{ \pm}}^{2}\right), \\
\lambda_{2} & =\frac{1}{2 v^{2}}\left(m_{H_{1}}^{2}-m_{A_{1}}^{2}\right), \\
\lambda_{23} & =g_{H_{1} H_{1} h}-\frac{2}{v^{2}}\left(m_{H_{1}}^{2}-m_{H_{1}^{ \pm}}^{2}\right),
\end{aligned}
$$

where $g_{H_{1} H_{1} h}=\lambda_{23}+\lambda_{23}^{\prime}+2 \lambda_{2}$ is the Higgs-DM coupling. The Feynman rules for this model are presented in appendix $\mathrm{A}$.

\section{Calculating the relic density in the $\mathrm{I}(2+1) \mathrm{HDM}$}

The relic density of the WIMP (identified in our model as the lightest inert scalar $H_{1}$ ) is calculated with the assumption that the WIMP was in thermal equilibrium with the SM particles after inflation. Once the rate of

\section{$\mathrm{DM} \mathrm{DM} \leftrightarrow \mathrm{SM} \mathrm{SM}$}

reactions becomes smaller than the Hubble expansion rate of the Universe, the WIMP freezes out, i.e., drops out of the thermal equilibrium. After freeze-out the co-moving WIMP density remains essentially constant with the current value estimated by the Planck experiment to be the one already given in eq. (1.1).

As mentioned, in the $\mathrm{I}(2+1) \mathrm{HDM}$ one of the neutral inert (pseudo-)scalar particles play the role of the DM. The relic density of a (pseudo-) scalar DM candidate, $S$, after freeze-out is given by the solution to the Boltzmann equation:

$$
\frac{d n_{S}}{d t}=-3 H n_{S}-\left\langle\sigma_{\mathrm{eff}} v\right\rangle\left(n_{S}^{2}-n_{S}^{e q}{ }^{2}\right), \quad S=H_{1}, H_{2}, A_{1}, A_{2}
$$

where the thermally averaged effective (co)annihilation cross-section contains all relevant scattering processes of any $S_{i} S_{j}$ pair into SM particles:

$$
\left\langle\sigma_{\mathrm{eff}} v\right\rangle=\sum_{i j}\left\langle\sigma_{i j} v_{i j}\right\rangle \frac{n_{i}^{e q}}{n_{S}^{e q}} \frac{n_{j}^{e q}}{n_{S}^{e q}},
$$

where

$$
\frac{n_{i}^{e q}}{n_{S}^{e q}} \sim \exp \left(-\frac{m_{i}-m_{S}}{T}\right)
$$

Therefore, only processes in which the mass splitting between a state $S_{i}$ and the lightest $Z_{2}$-odd particle $S$ ( $H_{1}$ in our case) are comparable to the thermal bath temperature $T$ provide a sizeable contribution to this sum.

In the $\mathrm{I}(2+1) \mathrm{HDM}$, the presence of additional inert particles has important consequences in the heavy mass regime. For lighter masses the most important channel for the annihilation of DM particles is the Higgs-mediated process

$$
H_{1} H_{1} \rightarrow f \bar{f}
$$


(see figure 12a), as studied in [14]. However, coannhilation with $H_{2}, A_{1}$ and $A_{2}$ may change the results significantly (see figure 12b).

For heavier masses the diagrams including one or two virtual gauge bosons, shown in figure 13 also contribute to the total annihilation cross-section. Finally, co-annihilation plays an important role in scenarios with multiple particles which are close in mass. This scenario is realised in the $\mathrm{I}(2+1) \mathrm{HDM}$ for the heavy DM mass region. Particles up to $20 \%$ heavier than the DM candidate may influence the DM relic density. Therefore, the co-annihilation diagrams should be included in calculating the effective annihilation crosssection. These diagrams are presented in figures 14 and 15 - representing pure gauge channels and coannhilation channels involving the SM-like Higgs particle, respectively.

\subsection{Relevant co-annihilation scenarios}

In the $\mathrm{I}(2+1) \mathrm{HDM}$, the strength and importance of coannhilation processes depend on the mass splittings between the inert particles. We define $\delta_{A}$ and $\delta_{C}$ as the splitting between $H_{1}$ and the pseudoscalar and charged state from the first generation, respectively,

$$
\delta_{A}=m_{A_{1}}-m_{H_{1}}, \quad \delta_{C}=m_{H_{1}^{ \pm}}-m_{H_{1}} .
$$

$\delta_{A, C}$ are related to the quartic couplings in the potential, which are constrained by the perturbativity (and unitarity) conditions, i.e., the $\lambda_{i}$ 's cannot be too large. As a result of this, all particles within one generation will have a similar mass. These masses, however, could have high values because of the (almost) unconstrained quadratic parameters $\mu_{2}^{2}$ and $\mu_{12}^{2}$ :

$$
m_{H_{1}}^{2}=-\mu_{2}^{2}-\mu_{12}^{2}+\frac{v^{2}}{2} g_{H_{1} H_{1} h} .
$$

It is important to stress that, even if bounds on $\lambda_{i}$ were relaxed leading to larger values of $\delta_{A, C}$, there exist very stringent limits from relic density analysis. Coannihilation must occur at least between $H_{1}, A_{1}$ and $H^{ \pm}$to achieve DM relic density in agreement with the current experimental measurements. This is a pattern followed by all general heavy scalar DM models. In the absence of these co-annihilation channels, the maximum relic density that can be achieved through $H_{1} H_{1} \rightarrow \mathrm{SM} \mathrm{SM}$ (even when $H_{i} H_{j} \rightarrow \mathrm{SM} \mathrm{SM}$ is allowed) is of order $10^{-3}$ which is well below the observed value.

The other important mass splitting, $\Delta$, is defined as the mass difference between $H_{1}$ and the other CP-even state $H_{2}$ ("splitting between doublets"):

$$
\Delta=m_{H_{2}}-m_{H_{1}}
$$

$\Delta$ is related to the quadratic parameter $\mu_{12}^{2}$ through

$$
\mu_{12}^{2}=\frac{1}{2}\left(m_{H_{2}}^{2}-m_{H_{1}}^{2}\right)=\frac{1}{2}\left(\Delta^{2}+2 m_{H_{1}} \Delta\right) .
$$

Note that $\mu_{12}^{2}$ is not limited by any theoretical constraints - similar to $\mu_{2}^{2}$ - and therefore $\Delta$ can in principle be very large. So, unless $\Delta$ is forced to be small by limits put on $\mu_{12}^{2}$, one should also consider a case where the second doublet is decoupled from the first, leading to a scenario which was not listed in [14]. Therefore, in the very heavy mass region one can consider: 


\begin{tabular}{|l|c|l|}
\hline & Small $\delta_{A}, \delta_{C}$ & Large $\delta_{A}, \delta_{C}$ \\
\hline Small $\Delta$ & Case $\mathrm{G}$ is realised & $\begin{array}{l}\text { Coannhilation between } H_{1}, H_{2} \text { is not efficient } \\
\text { enough and DM density is below experimental } \\
\text { bounds. }\end{array}$ \\
\hline Large $\Delta$ & Case $\mathrm{H}$ is realised & $\begin{array}{l}\text { There are no co-annihilatin channels open and } \\
\text { gauge annihlation reduces DM relic density ef- } \\
\text { fectively below the experimental bounds. }\end{array}$ \\
\hline
\end{tabular}

Table 1. Valid regions of the parameter space schematically shown in terms of $\delta_{A}, \delta_{C}$ and $\Delta$ in the heavy mass regime of $\mathrm{I}(2+1) \mathrm{HDM}$.

- Case G: with small $\delta_{A}, \delta_{C}, \Delta$, where all inert particles are close in mass and coannihilate with each other.

- Case $\mathbf{H}$ : with small $\delta_{A}, \delta_{C}$ and large $\Delta$, where the second generation is effectively decoupled from the first generation and does not influence relic density calculations. In this case, the relevant diagrams are the (co)annihilation channels between fields from the lighter generation only, $H_{1}, A_{1}, H_{1}^{ \pm}$.

Table 1 summarises the two possible scenarios for relic density studies.

\subsection{The gauge limit}

To illustrate the difference between cases $\mathrm{G}$ and $\mathrm{H}$, let us first consider the gauge limit in both scenarios, which is the limit where all quartic couplings $\lambda_{i}$ are set to zero. Therefore, all scalar self-couplings, including the DM-Higgs coupling, are removed in this limit. As a result $\delta_{A, C}=0$, leading to degenerate $H_{1}, A_{1}, H_{1}^{ \pm}$states. Note that this limit is excluded by results of direct detection experiments, nevertheless, it is an interesting limit to study as it represents the main difference between cases $\mathrm{G}$ and $\mathrm{H}$. In this limit $H_{1}$ annihilates solely through the gauge annihilation channels presented in figure 14 .

Non-zero $\lambda_{i}$ will lift this degeneracy and, at the same time, reduce the effective annihilation cross-section for a given mass. Therefore, the gauge limit corresponds to the minimum value of $m_{H_{1}}$, for which it is possible to obtain a proper relic density for any value of the Higgs-DM coupling.

These results are presented in figure 1 for the two scenarios: case $\mathrm{G}$ where all particles have degenerate mass (in the $\delta_{A, C}, \Delta \rightarrow 0 \Leftrightarrow \lambda_{i} \rightarrow 0, \mu_{12}^{2} \rightarrow 0$ limit) and case $\mathrm{H}$ with large $\Delta$ where the second generation is decoupled from the first generation and all particles in the first generation are degenerate in mass (in the $\delta_{A, C} \rightarrow 0 \Leftrightarrow \lambda_{i} \rightarrow 0$ limit).

It is clear that, for a given mass of $m_{H_{1}}$ the destructive interference between an increased number of coannhilation diagrams in case $\mathrm{G}$ leads to a reduced cross-section, i.e. larger DM relic density with respect to case $\mathrm{H}$. The important consequence of this interference is that for case $\mathrm{G}$ it is possible to obtain proper relic density for smaller masses of DM candidate in comparison to case $\mathrm{H}$. Note also that case $\mathrm{H}$ behaves like the $\mathrm{I}(1+1) \mathrm{HDM}$ (the Inert Doublet Model) in this limit. This similarity in behaviour will be repeated as we will show in the following sections. 


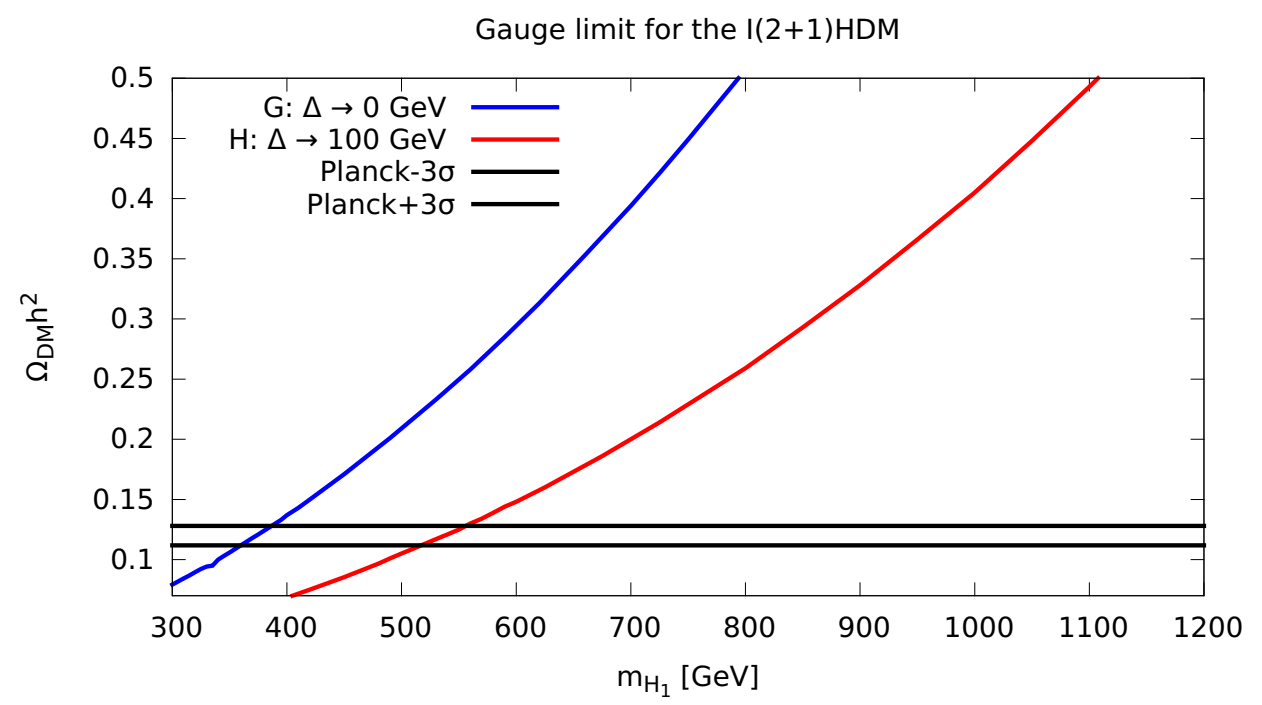

Figure 1. The gauge limit in case $\mathrm{G}$ (blue curve) where all particles have degenerate mass (in the $\delta_{A, C}, \Delta \rightarrow 0$ ) and case $\mathrm{H}$ (red curve) with large $\Delta$ where the second generation is decoupled from the first generation and all particles in the first generation are degenerate in mass (in the $\delta_{A, C} \rightarrow 0$ limit).

\subsection{The benchmark points}

With non-zero scalar couplings and mass splitting more diagrams contribute to the coannihilation of DM - all diagrams shown in figures 14 and 15 contribute to the total annihilation cross-section. Here we present two benchmarks points, two sets of parameters, for which we have studied the DM relic density:

- For case $\mathrm{G}$ with $\delta_{A}=\delta_{C}=1 \mathrm{GeV}$ and $\Delta=1 \mathrm{GeV}$

Here all inert particles have similar masses and therefore can co-annihilate with each other. The degeneracy between charged and "pseudo-scalar" particles is allowed and doesn't lead to any unacceptable results. The important degeneracy which must be avoided is $H_{1}-A_{1}$ degeneracy leading to the scattering through the $Z$ boson which is tightly constrained by direct detection experiments and puts a lower limit on $\delta_{A}$.

- For case $\mathbf{H}$ with $\delta_{A}=\delta_{C}=1 \mathrm{GeV}$ and $\Delta=100 \mathrm{GeV}$

Here the second generation of inert scalars is significantly heavier than the first one. Within each generation, however, particles are almost degenerate.

Note that there are certain differences between cases $\mathrm{G}$ and $\mathrm{H}$. In case $\mathrm{H}$, the heavier generation of inert particles is decoupled from the first generation particles and does not influence the relic density calculations. The model in this case resembles the I(1+1)HDM. Furthermore, in case G the Higgs-DM couplings which result in a relic density in agreement with experiment are larger in comparison to case $\mathrm{H}$ for the same DM mass. This difference is explicit in figure 2 . 


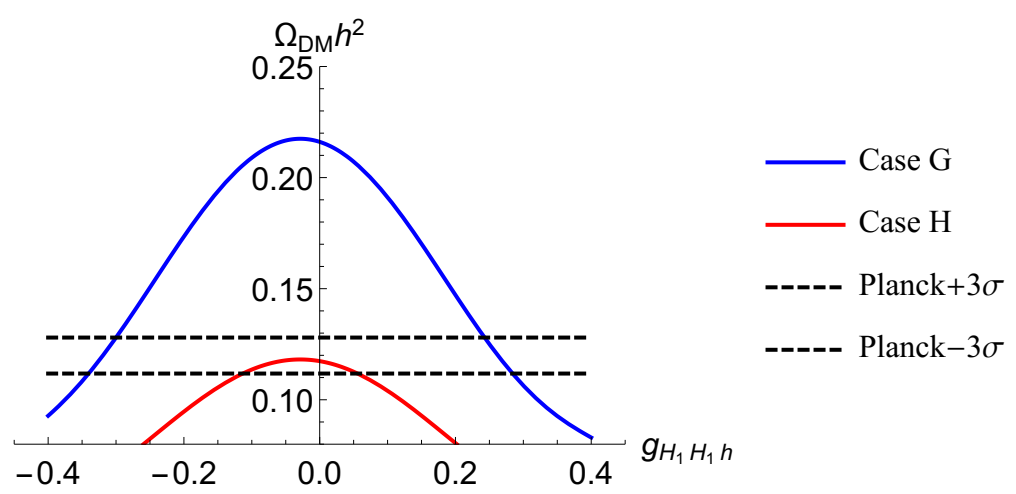

Figure 2. Relic density plots in case $\mathrm{G}(\Delta=1 \mathrm{GeV}$, blue line) and $\mathrm{H}(\Delta=100 \mathrm{GeV}$, red line $)$ for $m_{H_{1}}=550 \mathrm{GeV}$. The dashed horizontal lines show the $3 \sigma$ relic density limits from Planck in eq. (1.1).
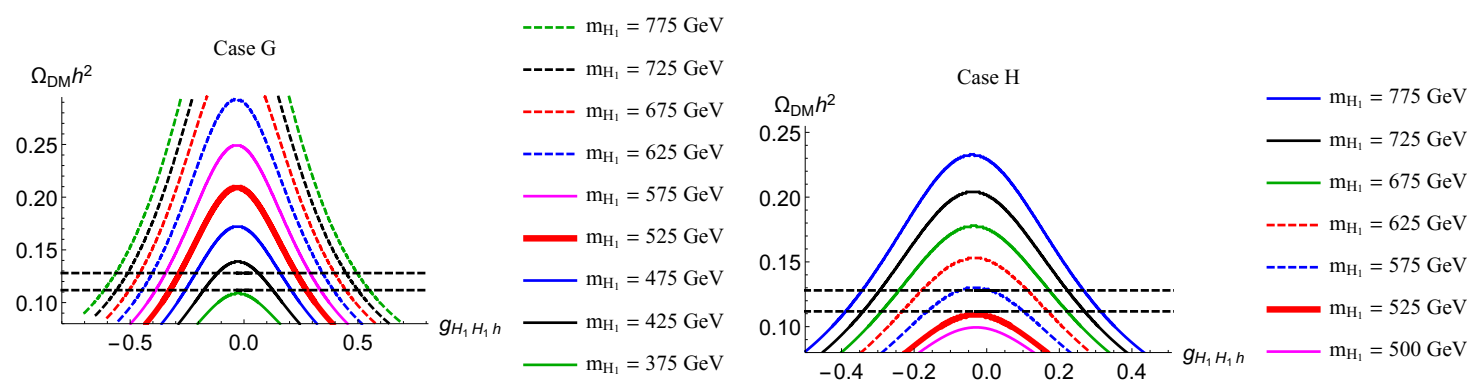

Figure 3. Relic density plots for case $\mathrm{G}$ (left) with $\Delta=1 \mathrm{GeV}, \delta=1 \mathrm{GeV}$ and case $\mathrm{H}$ (right) with $\Delta=100 \mathrm{GeV}, \delta=1 \mathrm{GeV}$. Note the solid red line in the case $\mathrm{H}$ which represents the minimum DM mass $m_{H_{1}}=525 \mathrm{GeV}$ which just touching the lower relic density limit. The relic density plot for the same DM mass has been highlighted in case G (red solid line) which is well within the acceptable relic density limits. The dashed horizontal lines show the $3 \sigma$ relic density limits from Planck in eq. (1.1).

This leads to the fact that for case $\mathrm{G}$ we can obtain viable relic density values for $m_{\mathrm{DM}}$ much smaller than in case $\mathrm{H}$ (or the $\mathrm{I}(1+1) \mathrm{HDM}$ ) in which the minimal value of $m_{\mathrm{DM}}$ resulting in DM relic density in agreement with Planck limits is $m_{H_{1}} \approx 525-535 \mathrm{GeV}$. In case $\mathrm{G}$ (with $\Delta=1 \mathrm{GeV}$ ), however, the DM mass can be as low as $\sim 375 \mathrm{GeV}$. This result is shown in figure 3 which represents relic density plots for cases $\mathrm{G}$ (left) and $\mathrm{H}$ (right). Note that in case $\mathrm{G}$ the minimum $m_{H_{1}}$ which touches the lowest acceptable relic density limit (the green solid line) is $375 \mathrm{GeV}$ (for a given $\Delta$ of $1 \mathrm{GeV}$ ), whereas in case $\mathrm{H}$ this minimum value is $525 \mathrm{GeV}$ (the solid red line).

Figure 4 is meant to represent the same benchmark points as in figure 3 , in the $m_{H_{1}}$ $g_{H_{1} H_{1} h}$ plane. The bands correspond to proper relic density in agreement with Planck measurements in case $\mathrm{G}$ (for an exemplary $\Delta=1 \mathrm{GeV}, \delta=1 \mathrm{GeV}$ ) in red and case $\mathrm{H}$ (for an exemplary $\Delta=100 \mathrm{GeV}, \delta=1 \mathrm{GeV}$ ) in red. Note that, for the a given DM mass (and same $\delta$ ), the Higgs-DM coupling in case $\mathrm{G}$ is much larger than in case $\mathrm{H}$. 


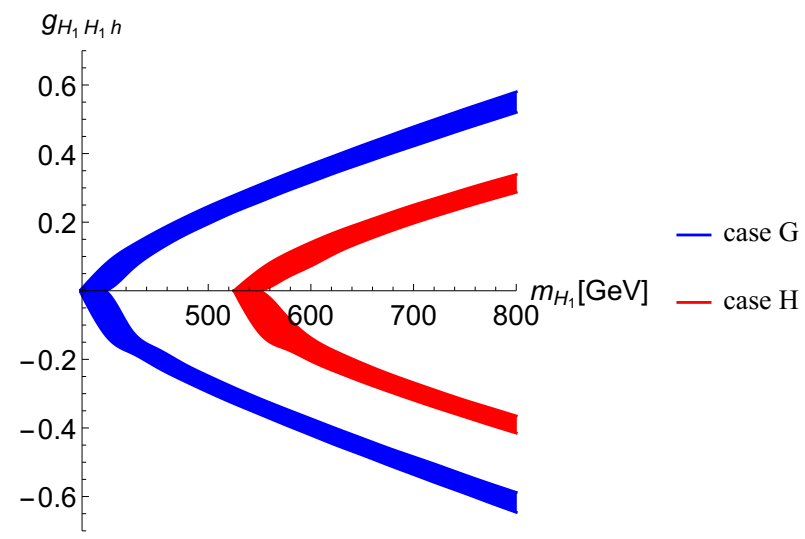

Figure 4. Relic density bands in agreement with Planck measurements in case $\mathrm{G}$ (for an exemplary $\Delta=1 \mathrm{GeV}, \delta=1 \mathrm{GeV}$ ) in red and case $\mathrm{H}$ (for an exemplary $\Delta=100 \mathrm{GeV}, \delta=1 \mathrm{GeV}$ ) in red. Note that for the a given DM mass, the Higgs-DM coupling in case G is much larger than in case H.

\section{DM in the $\mathrm{I}(2+1) \mathrm{HDM}$ : direct detection}

\subsection{Changes in $\Delta, \delta_{A}, \delta_{C}$}

For the two viable scenarios $\mathrm{G}$ and $\mathrm{H}$ we allow the physical parameters to vary in the following regions:

$$
100 \mathrm{keV} \lesssim \delta_{A}, \delta_{C} \lesssim 15 \mathrm{GeV} .
$$

Since $\delta_{A}$ and $\delta_{C}$ are related to the quartic parameters $\lambda_{i}$ 's, they are constrained from unitarity bounds and are required to be small. However, regardless of any limits on $\lambda_{i}$ from unitarity, relic density studies show that both $\delta_{A}$ and $\delta_{C}$ must be relatively small to allow for co-annihilation between particles which is crucial in the heavy DM mass region. The upper limit follows the following rough rule: co-annihilation effects take place when the mass difference between co-annihilating particles is of the order of $20 \%$ of their mass.

The lower bound on $\delta_{A}$ comes from direct detection experiments where a degeneracy between $H_{1}$ and $A_{1}$ leads to the scattering through the $Z$ boson which is tightly constrained. Further, the above limits in eq. (4.1) are in agreement with LEP searches for exotic particles. Finally, below $\delta_{A, C} \sim 0.1 \mathrm{GeV}$ there is no visible difference in the results.

As mentioned before, a large difference between $\delta_{A}$ and $\delta_{C}$ violates relic density limits. In the cases we study below, we have set $\delta_{A}=\delta_{C}=\delta$ for simplicity. ${ }^{5}$ The mass splitting between the two generations, $\Delta$, is proportional to and therefore unconstrained by unitarity. The maximum value for $\Delta$ is proportional to the arbitrary maximum value chosen for $\mu_{12}^{2}$. In general, we allow for $\Delta$ to vary in the following region

$$
100 \mathrm{keV} \lesssim \Delta \lesssim 200 \mathrm{GeV}
$$

For large $\Delta$ values, $\Delta \gtrsim 20-50 \mathrm{GeV}$ (depending on $m_{H_{1}}$, since the story-changing mass splitting is roughly $20 \% m_{H_{1}}$ ), co-annihilation effects between the two generations

\footnotetext{
${ }^{5}$ Cases with $\delta_{A} \neq \delta_{C}$ do not lead to any new phenomenology and in fact the region of validity for $g_{H_{1} H_{1} h}$ decreases as we increase the difference between $\delta_{A}$ and $\delta_{C}$.
} 
are not strong enough to compete with the standard (co)annhilation between $H_{1}, A_{1}, H_{1}^{ \pm}$, in which case the $\mathrm{I}(2+1) \mathrm{HDM}$ acts just like the $\mathrm{I}(1+1) \mathrm{HDM}$. The second generation is effectively decoupled from the first generation and does not influence DM phenomenology. Therefore, scenario $\mathrm{H}$ is realised for:

$$
\Delta \gtrsim 20 \mathrm{GeV} \Rightarrow \text { scenario } \mathrm{H}
$$

The exact value of $\Delta$, when above $\sim 20-50 \mathrm{GeV}$ does not make any significant difference in the relic density calculations.

For small values of $\Delta$, the co-annihilation effects between all particles are important. The smaller $\Delta$ is, the more relevant particles from the second generation are for DM studies: for $\Delta \approx 1.5 \mathrm{GeV}$ the relative contribution to relic density calculation coming from particles from the lighter generation to the heavier generation is $70 \%-30 \%$. For $\Delta \approx 0.0001 \mathrm{GeV}$ this relation is $50 \%-50 \%$. The case $\mathrm{G}$, is therefore realised when $\Delta$ varies in the following window

$$
0.0001 \mathrm{GeV} \lesssim \Delta \lesssim 20 \mathrm{GeV} \Rightarrow \text { scenario } \mathrm{G}
$$

The closer $\Delta$ gets to this upper limit, the weaker the coannnihilation effects and the more scenario $\mathrm{H}$ is realised. Finally, notice that, for our studies, the Higgs-DM coupling, $g_{h H_{1} H_{1}}$, is kept within the $\left|g_{h H_{1} H_{1}}\right|<1$ range.

Figure 5 illustrates the effect of changing $\Delta$ on the relic density. In all four plots $m_{\mathrm{DM}}$ has been set to $400 \mathrm{GeV}$ as the value of $\Delta$ changes, $0.1 \mathrm{GeV}$ in the top left plot, $1 \mathrm{GeV}$ in the top right plot, $5 \mathrm{GeV}$ in the bottom left plot and $10 \mathrm{GeV}$ in the bottom right plot. In each plot different colours represent different $\delta$ s. For small values of $\Delta(0.1 \mathrm{GeV})$, the $H_{1^{-}}$ $H_{2}$ co-annihilation leads to viable relic density values even for large $\delta$ (i.e. the $H_{1}, A_{1}, H^{ \pm}$ co-annihilation is absent). For large values of $\Delta(10 \mathrm{GeV}) H_{1}-H_{2}$ co-annihilation does not exist and even small values of $\delta$ cannot compensate this lack, thus, the relic density is below the acceptable limit.

In figure 6 the value of $\delta$ is set to $0.5 \mathrm{GeV}$ for $m_{\mathrm{DM}}=400 \mathrm{GeV}$ in the left plot and $m_{\mathrm{DM}}=550 \mathrm{GeV}$ in the right plot. In each plot, different colours correspond to changing $\Delta \mathrm{s}$. Note that in the left plot only small values of $\Delta$ lead to viable relic density values, which is where case $\mathrm{G}$ is realised. In the right plot, small values of $\Delta$ correspond to case $\mathrm{G}$ and large values of $\Delta$ correspond to case $\mathrm{H}$, and they all lead to acceptable relic density values. Note that for $\Delta \gtrsim 50 \mathrm{GeV}$ all curves correspond to the same value.

\subsection{Changes in $m_{\mathrm{DM}}$}

Here we describe several sub-regimes where the DM mass can vary with important characteristics.

- In the region

$$
m_{\mathrm{DM}} \lesssim 360 \mathrm{GeV}
$$

neither scenario $\mathrm{H}$ nor $\mathrm{G}$ results in viable relic density values. ${ }^{6}$ This lower limit can be reached in case $G$ by very specific points in the parameter space: (a) when the

\footnotetext{
${ }^{6}$ In extensions of the $\mathrm{I}(2+1) \mathrm{HDM}$ with more inert doublets, this $m_{\mathrm{DM}}$ limit could be lowered as more co-annihilation channels are present due to the extended number of inert particles.
} 

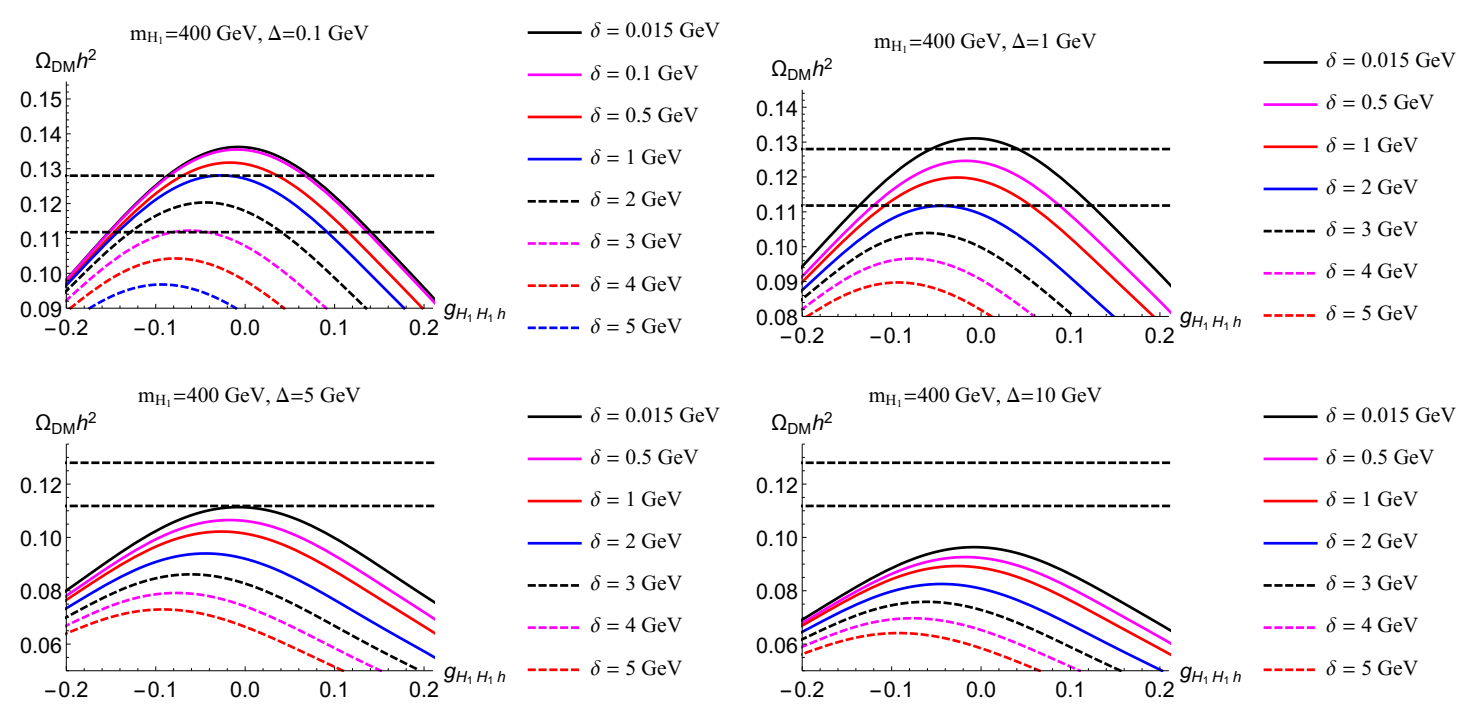

Figure 5. The effect of changing $\Delta$ on the relic density. In all four plots $m_{\mathrm{DM}}$ has been set to $400 \mathrm{GeV}$ as the value of $\Delta$ changes, $0.1 \mathrm{GeV}$ in the top left plot, $1 \mathrm{GeV}$ in the top right plot, $5 \mathrm{GeV}$ in the bottom left plot and $10 \mathrm{GeV}$ in the bottom right plot. In each plot different colours represent different $\delta \mathrm{s}$. For small values of $\Delta(0.1 \mathrm{GeV})$, the $H_{1}-H_{2}$ co-annihilation leads to viable relic density values even for large $\delta$ (i.e. the $H_{1}, A_{1}, H^{ \pm}$co-annihilation is absent). For large values of $\Delta(10 \mathrm{GeV}) H_{1}-H_{2}$ co-annihilation does not exist and even small values of $\delta$ cannot compensate this lack, thus, the relic density is below the acceptable limit.

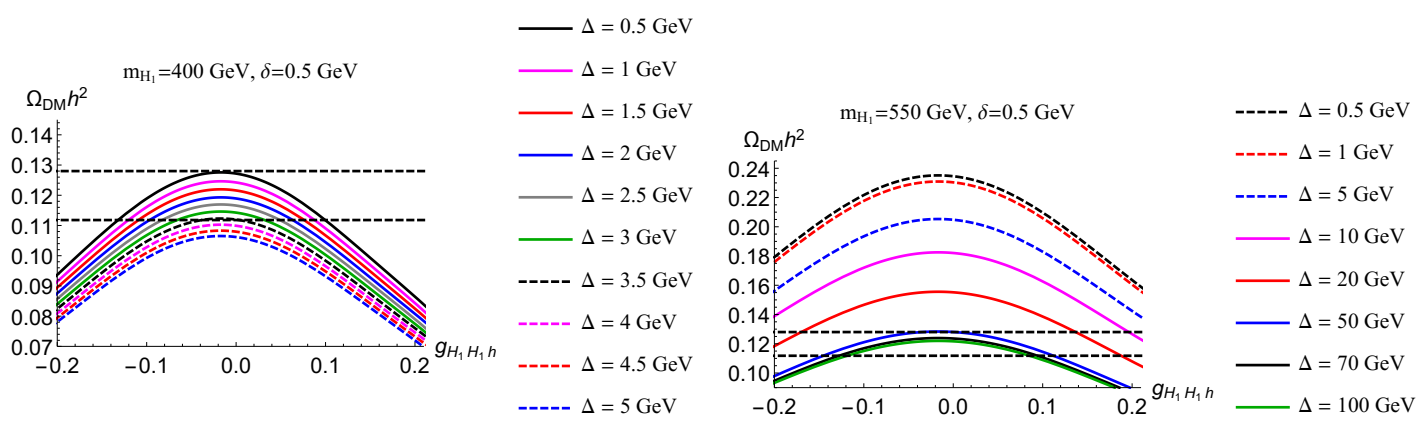

Figure 6. Relic density plot for $\delta=0.5 \mathrm{GeV}$ for $m_{\mathrm{DM}}=400 \mathrm{GeV}$ in the left plot and $m_{\mathrm{DM}}=$ $550 \mathrm{GeV}$ in the right plot. In each plot, different colours correspond to changing $\Delta \mathrm{s}$. Note that in the left plot only small values of $\Delta$ lead to viable relic density values, which is where case $\mathrm{G}$ is realised. In the right plot, small values of $\Delta$ correspond to case $\mathrm{G}$ and large values of $\Delta$ correspond to case $\mathrm{H}$, and they all lead to acceptable relic density values. Note that for $\Delta \gtrsim 50 \mathrm{GeV}$ all curves correspond to the same value.

mass splitting between all particles is tiny and all particles are almost degenerate in mass (up to $\mathcal{O}(100 \mathrm{keV}$ ) mass splitting to avoid direct detection limits), (b) when $g_{h H_{1} H_{1}}$ is close to 0 .

- In the region

$$
360 \mathrm{GeV} \lesssim m_{\mathrm{DM}} \lesssim 525 \mathrm{GeV}
$$




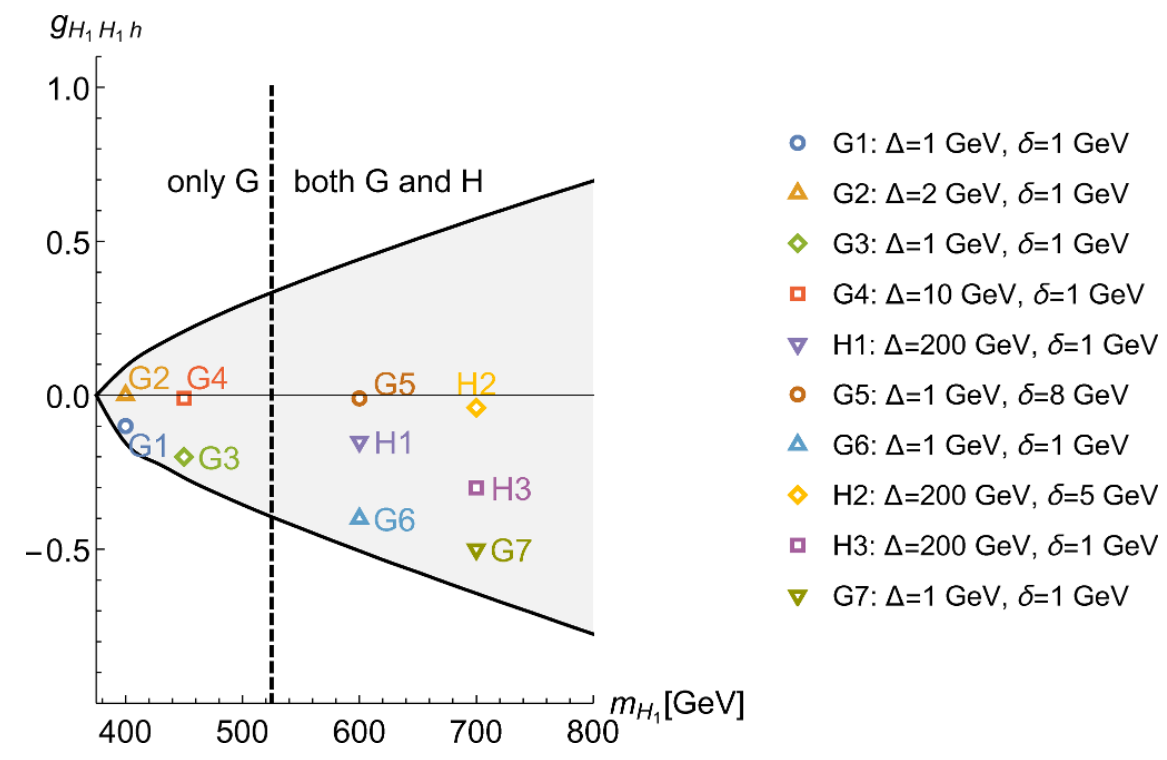

Figure 7. Here $\mathrm{G} i$ and $\mathrm{H} i$ represent certain points in the parameter space corresponding to cases $\mathrm{G}$ and $\mathrm{H}$, respectively. The shaded region is where the $\mathrm{I}(2+1) \mathrm{HDM}$ has acceptable relic density results. To the left of the vertical dashed line case $\mathrm{G}$ is realised and to the right of it both cases $\mathrm{G}$ and $\mathrm{H}$ are realised. Generally the outermost parts of the shaded region are populated by $\mathrm{G} i$ since they correspond to larger Higgs-DM couplings, and the innermost parts of the region correspond to case $\mathrm{H}$. However, depending on the values of $\delta$ the Higgs-DM coupling changes and Gi points can appear close to the $g_{h H_{1} H_{1}}=0$ line as well, which is apparent in comparing the points G5, H1 and G6. Other points are shown on the plot for different values of $\Delta$ and $\delta$ for comparison.

only scenario $\mathrm{G}$ leads to acceptable values of DM relic density for specific values of $\Delta$ in the $0.0001 \mathrm{GeV} \lesssim \Delta \lesssim 20 \mathrm{GeV}$ and $\left|g_{H_{1} H_{1} h}\right| \lesssim 0.3$ window. As a rule of thumb, smaller $\Delta$ allows for a wider viable region in the parameter space and the larger $m_{H_{1}}$ is the larger $\left|g_{h H_{1} H_{1}}\right|$ must be.

- In the region

$$
535 \mathrm{GeV} \lesssim m_{\mathrm{DM}} \lesssim 1.5-2 \mathrm{TeV}
$$

both scenarios, $\mathrm{G}$ and $\mathrm{H}$, lead to viable values of $\mathrm{DM}$ relic density. The appropriate value of $g_{H_{1} H_{1} h}$ coupling depends on the DM mass in each case. In scenario G, couplings are generally larger compared to scenario $\mathrm{H}$ and a larger DM mass requires larger values of the $g_{H_{1} H_{1} h}$ coupling.

Figure 7 is meant to summarise all that was said above in one plot with $\mathrm{G} i$ and $\mathrm{H} i$ representing certain points in the parameter space corresponding to cases $\mathrm{G}$ and $\mathrm{H}$, respectively. The shaded region is where the $\mathrm{I}(2+1) \mathrm{HDM}$ has acceptable relic density results. To the left of the vertical dashed line case $\mathrm{G}$ is realised and to the right of it both cases $\mathrm{G}$ and $\mathrm{H}$ are realised. Generally the outermost parts of the shaded region are populated by $\mathrm{G} i$ since they correspond to larger Higgs-DM couplings whereas the innermost parts of the region correspond to case H. However, depending on the values of $\delta$ the HiggsDM coupling changes and Gi points can appear close to the $g_{h H_{1} H_{1}}=0$ line as well, which 


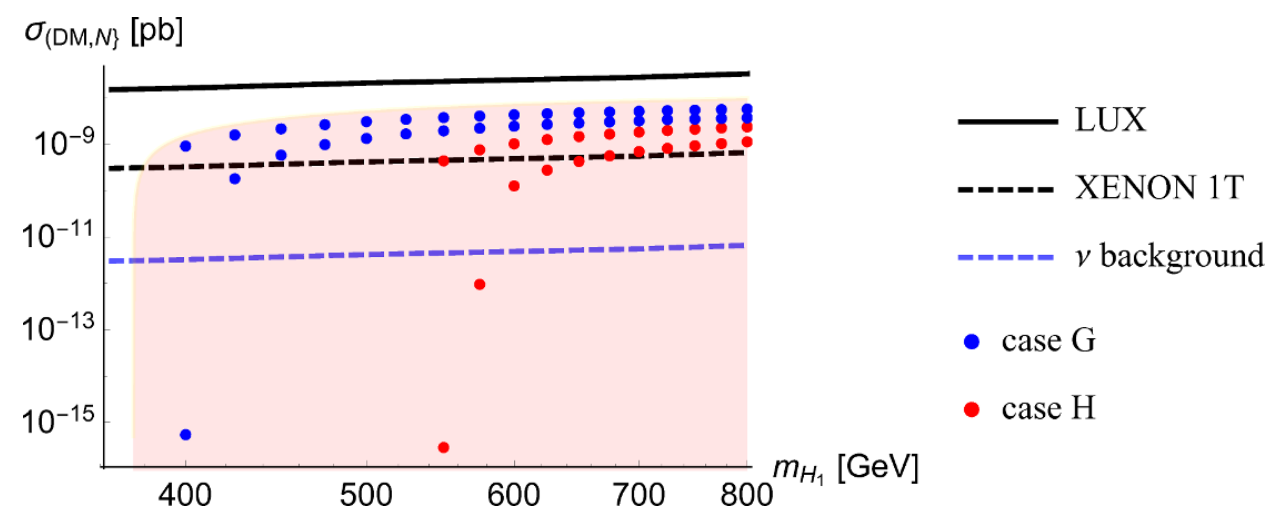

Figure 8. The DM-nucleon scattering cross-section for the $\mathrm{I}(2+1) \mathrm{HDM}$ in comparison with the direct detection limits set by LUX (black line) and projected XENON 1T (black dashed line). Coherent neutrino scattering limit is also shown (dashed blue line). The shaded region corresponds to points with relic density in agreement with Planck measurements; results for benchmark points for case $\mathrm{G}(\Delta=1 \mathrm{GeV}, \delta=1 \mathrm{GeV}$, blue) and case $\mathrm{H}(\Delta=100 \mathrm{GeV}, \delta=1 \mathrm{GeV})$ are presented.

is apparent in comparing the points G5, H1 and G6. Other points are shown on the plot for different values of $\Delta$ and $\delta$ for comparison.

\subsection{Direct detection}

Direct detection experiments, which are mostly designed to hunt for the standard EWscale WIMP, are the most sensitive to DM masses of the order of $100 \mathrm{GeV}$. For heavier DM masses the sensitivity of these experiments drops significantly. The most recent LUX results set the limit of the DM-nucleon scattering cross-section to be $\sigma_{\mathrm{DM}-N} \approx 10^{-8} \mathrm{pb}$ for DM masses $\approx 500-1000 \mathrm{GeV}[18]$.

In the $\mathrm{I}(2+1) \mathrm{HDM}$, similar to the other scalar DM models, DM candidate can be detected through elastic scattering on nuclei through the exchange of a Higgs particle. Therefore, $\sigma_{\mathrm{DM}-N}$ will depend on the value of DM-Higgs coupling, and the DM mass.

These results are presented in figure 8 where the shaded region corresponds to the probed phase space of the I $(2+1) \mathrm{HDM}$ for various choices of $\Delta$ and $\delta$ (as shown in figure 7 ), all of which have relic density in agreement with Planck measurements. Also, results for the benchmark points studied in section 3 are presented explicitly. We found that the current experimental limits do not constrain the heavy DM mass region, neither for case $\mathrm{H}$ nor for case $\mathrm{G}$, even though the Higgs-DM coupling is larger in the latter case.

Recall that for certain choices of $\Delta$ and $\delta$ one can obtain proper DM relic density for $g_{H_{1} H_{1} h} \approx 0$. This leads to a strongly suppressed scattering cross-section, which may not be detected as it lies within the coherent neutrino-nucleus scattering regime [19].

Figure 8 also shows a limit from the future XENON1T experiment [20], with a proposed sensitivity of the order of $10^{-9}-10^{-10} \mathrm{pb}$ (dashed black line). We expect the next generation of DM detectors, such as XENON1T, to be able to test a large portion of the parameter space of the heavy DM in the I(2+1)HDM for $m_{H_{1}} \lesssim 1 \mathrm{TeV}$. 
As a final note to this subsection, we should like to mention that a viable intermediate DM mass region, $m_{W} \lesssim m_{\mathrm{DM}} \lesssim 160 \mathrm{GeV}$, regarding relic density studies, has been found in the $\mathrm{I}(1+1) H D M$. The correct relic density in this region is obtained due to cancellations between different diagrams contributing to DM annihilation into gauge bosons $\left(W^{+} W^{-}\right.$ and $Z Z$ ). In [21] it was shown that this scenario is realised if the inert particles, in particular the charged scalar, are heavy enough, $\sim 300-500 \mathrm{GeV}$. A relatively large DMHiggs coupling is also required for the DM in this mass region to stay within relic density limits, however, this large Higgs-DM coupling is excluded by LUX. Similarly, we did not find any solutions in the medium mass region with viable relic density and in agreement with direct detection experiments in the I $(2+1) \mathrm{HDM}$.

\section{Heavy Higgs DM at the LHC in the I(2+1)HDM}

A scalar DM candidate is a stable particle with limited interactions with all SM particles and therefore it cannot be directly detected at the LHC. However, its presence can influence the detectable properties of SM particles. One way to ascertain the influence of DM candidate on the properties of a Higgs particle is to look at the Higgs invisible decays, $h \rightarrow S S$, where $S$ is a scalar DM candidate with mass below $m_{h} / 2$. Invisible decays of the SM-like Higgs particle in the I $(2+1)$ HDM were studied in [14, 22], where limits for the mass of a light DM candidate combined with Planck limits for the relic density measurements provided constraints comparable or stronger than those from direct detection experiments.

Another strategy, useful for a heavy DM particle, is to look for a high $p_{T}$ monojet or a two jet/two lepton signal, accompanied by a large missing transverse energy $\mathscr{E}_{T}$. The monojet signature in the I( $2+1) \mathrm{HDM}, p p \rightarrow H_{1} H_{1}+$ jet, corresponds to $h$ coupling to an invisible pair of DM particles (yielding the large $E_{T}$ ) with produced in association with an energetic quark or gluon jet. The following processes are considered in our analysis.

1. $g g \rightarrow g H_{1} H_{1}$ (figure 16) via a triple gluon and a $h g g$ effective vertex. Note, that the $h g g$ effective vertex in the $\mathrm{I}(2+1) \mathrm{HDM}$ is the same as in the SM, as the Higgs production here is not modified by presence of additional scalar states. This is the dominant contribution to the monojet process, as the gluon fusion is an enhanced production mechanism for the Higgs particle.

2. $q \bar{q} \rightarrow g H_{1} H_{1}$ (figure 17), where $q=u, d, c, s, b$. The dominant contribution comes from the $s$-channel via the $g q \bar{q}$ tree-level vertex and the $h g g$ effective coupling (figure 17a).

3. $q g \rightarrow q H_{1} H_{1}$ (figure 18), where $q=u, d, c, s, b$. The dominant contributions here come from $g b \rightarrow H_{1} H_{1} b$ with the Higgs boson radiated off of the $b$ quark legs (figure 18a) and $q g \rightarrow q H_{1} H_{1} t$-channel via a $g q \bar{q}$ tree-level vertex and the $h g g$ effective coupling (figure 18b).

Note, that all above processes contain the $h \rightarrow H_{1} H_{1}$ vertex, therefore a strong dependency on the $g_{H_{1} H_{1} h}$ coupling is expected, i.e., a significant difference between scenarios $\mathrm{G}$ and $\mathrm{H}$, as discussed in the previous section. 
For the studies of $p p \rightarrow H_{1} H_{1}+2$ jets we have considered the Vector Boson Fusion (VBF) process of the form $q_{i} q_{j} \rightarrow H_{1} H_{1} q_{k} q_{l}$, with $q=u, d$ where a pair of DM particles (with large $E_{T}$ ) is produced by the neutral (figure 19) or charged (figure 20) VBF processes, either directly or mediated by the Higgs particle or another neutral scalar.

We have also considered the Higgs-Strahlung (HS) processes of the form $q_{i} \overline{q_{j}} \rightarrow$ $V^{*} H_{1} H_{1}$ where a pair of DM particles is radiated off the $Z$ or $W^{ \pm}$boson leg (figure 21 and figure 22, respectively), either directly or mediated by the Higgs particle or another neutral scalar.

Notice, that in the dijet searches only one diagram out of each set depends on the $g_{H_{1} H_{1} h}$, therefore we expect smaller differences between scenarios $\mathrm{H}$ and $\mathrm{G}$ than in the monojet searches. The strength of the other diagrams is set by the gauge interactions.

Also, it is important to stress that, given our initial choice of parameters, i.e. introducing the $k=1$ relation between the doublets (see eq. (2.11)), we have limited the number of possible diagrams, because vertices of the type $Z H_{i} A_{j}$ and $W^{ \pm} H_{i} H_{j}^{\mp}(i \neq j)$ are absent when $k=1$. Relaxing this initial assumption would in principle not only influence the evolution of DM relic density, but could also lead to a possibly stronger difference between scenarios $\mathrm{G}$ and $\mathrm{H}$ in the dijet analysis.

In the following subsections we present results for the monojet and dijet analysis, for the $14 \mathrm{TeV}$ LHC. The following selections were used.

1. For the monojet searches, we require the following cuts on the transverse momentum of the jet, $p_{T}^{j}$, and the pseudo-rapidity of the jet, $\eta^{j}$,

$$
p_{T}^{j}>20 \mathrm{GeV} \text { and }\left|\eta^{j}\right|>2.5
$$

2. For the dijet searches, we require the following cuts on the invariant mass of the two jets, $M(j, j)$, and the difference between the pseudo-rapidity of the forward and backward jet,

$$
M(j, j)>700 \mathrm{GeV} \text { and }\left|\eta_{f}^{j}-\eta_{b}^{j}\right|>4
$$

Calculations were done with the aid of LanHEP [23] and CalcHEP [24] packages.

\subsection{Monojet results}

In figure 9 results for monojet signals of scenarios $\mathrm{G}\left(\delta_{A}=\delta_{C}=1 \mathrm{GeV}, \Delta=1 \mathrm{GeV}\right)$ and $\mathrm{H}\left(\delta_{A}=\delta_{C}=1 \mathrm{GeV}, \Delta=100 \mathrm{GeV}\right)$ are shown. For comparison, we also present results for the I $(1+1) H D M$, with $\delta_{A}=\delta_{C}=1$ from [12]. The DM-Higgs coupling (defined as $\lambda_{345}$ in [12]) is the same as $g_{H_{1} H_{1} h}$ in scenario $\mathrm{H}$ for equal DM masses, therefore the monojet diagrams in case $\mathrm{H}$ and in $\mathrm{I}(1+1) \mathrm{HDM}$ are identical.

Scenario G, which corresponds to much larger Higgs-DM couplings compared to that of scenario $\mathrm{H}$ or the $\mathrm{I}(1+1) \mathrm{HDM}$, results in a significantly larger cross-section in the monojet process. Also the special features of the model are more visible in this process. For masses up to $m_{H_{1}} \approx 450 \mathrm{GeV}$ we observe a rise in the cross-section connected to an opening of the phase space combined with an increasing Higgs-DM coupling. After that peak, the cross-section decreases with increasing DM mass regardless of the rising of $g_{h H_{1} H_{1}}$. 


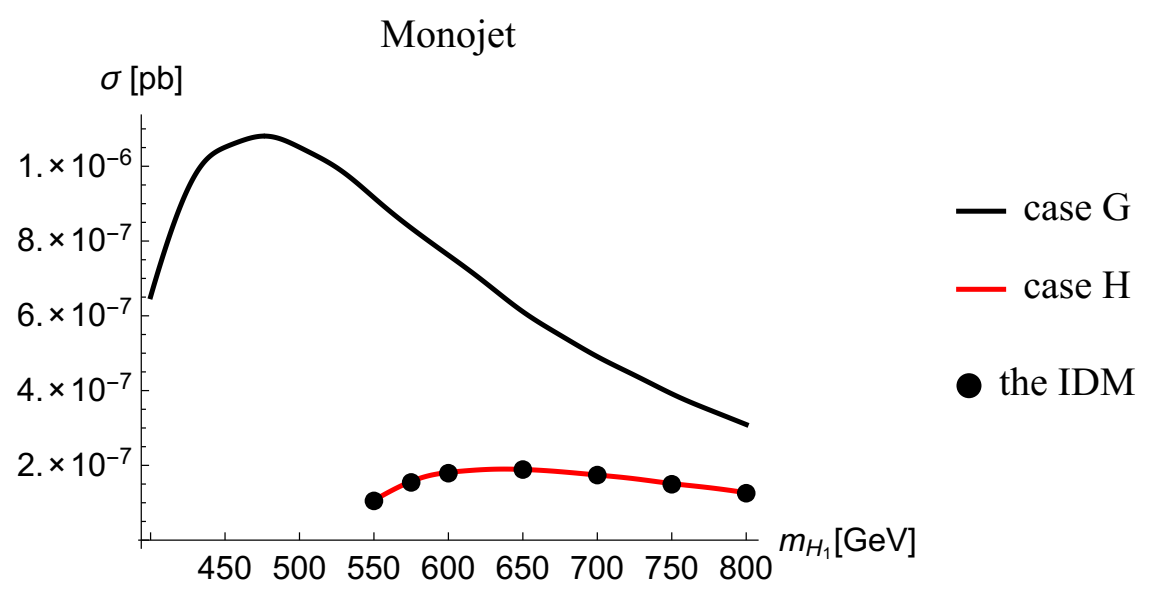

Figure 9. Monojet searches for cases $\mathrm{G}$ and $\mathrm{H}$. For comparison we also provide monojet searches for the $\mathrm{I}(1+1) \mathrm{HDM}$ which resemble case $\mathrm{H}$ in the $\mathrm{I}(2+1) \mathrm{HDM}$ as expected.

Notice that for the lower masses, the difference between scenario $\mathrm{G}$ and $\mathrm{H}$ is significant, as every diagram involved in the monojet process contains the $h H_{1} H_{1}$ vertex, whose coupling differs significantly in cases $\mathrm{G}$ and $\mathrm{H}$. Notice the low end of the allowed mass region in case $\mathrm{G}$ with a large cross-section for the mass region which is not even accessible by case $\mathrm{H}$ or the $\mathrm{I}(1+1) \mathrm{HDM}$. As the DM mass grows, results for both cases get closer together, stabilising for the very heavy mass region in the decoupling limit.

\subsection{Dijet VBF results}

Figure 10 presents values of the dijet cross-section for scenarios $\mathrm{H}$ and $\mathrm{G}$ in terms of the DM mass. The difference between cases $\mathrm{H}$ and $\mathrm{G}$ is less prominent compared to the monojet analysis, as only one of the involved diagrams in this process depends on the value of the Higgs-DM coupling (see figures 19 and 20 for diagrams involved in the neutral and charged VBF processes, respectively). We can still observe some differences in the lower mass range. The cross-section for case $\mathrm{G}$ is generally larger (as is the $g_{h H_{1} H_{1}}$ strength) than in case $\mathrm{H}$. Also, charged channels have slightly larger cross-sections than the neutral ones, since the cross-section for producing the $W^{ \pm}$boson is larger than the cross-section for producing the $Z$ boson. For the heavier masses all results, for both scenarios $\mathrm{G}$ and $\mathrm{H}$, as well as for charged and neutral channels, tend to converge.

\subsection{HS results}

HS signatures depend on the $W$ and $Z$ decay patterns. While at the LHC, leptonic signatures are preferred, hadronic ones are also possible. The latter potentially interfere with the VBF topologies, but the effect is small so that we can safely ignore it here.

The results of the (on-shell) HS process cross-sections in terms of the DM mass are presented in figure 11. It is clear that the general picture is different from the VBF studies. The largest cross-section again appears in the lower mass region where only case G can be realised. Similarly to the VBF case, the charged channels have larger cross- 


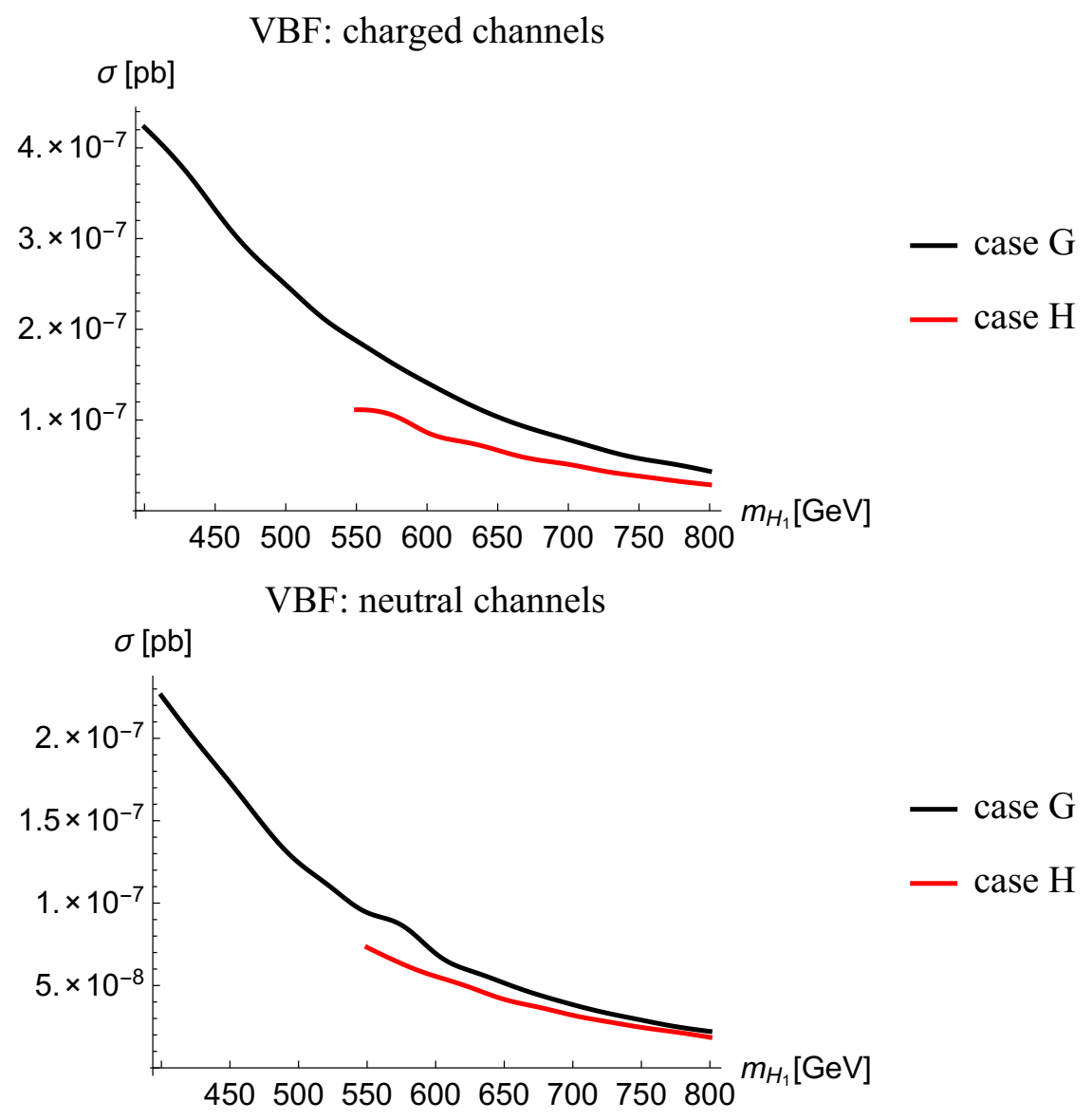

Figure 10. The cross-section in dijet VBF processes in terms of the DM mass in charged (top) and neutral (bottom) processes.

section compared to the neutral channels since the $W^{ \pm}$production in theses processes has a larger cross-section than the $Z$ boson production. All cross-sections in neutral and charged processes in both cases $\mathrm{G}$ and $\mathrm{H}$ converge to a similar value as $m_{\mathrm{DM}}$ approaches very large values.

Note that the cross-section in both $\mathrm{G}$ and $\mathrm{H}$ are similar in the region of DM mass above $525 \mathrm{GeV}$, i.e., where both cases can be realised (unlike in the VBF processes). This similarity is the result of the fact that the difference in the Higgs-DM coupling does not translate into a difference in the cross-section between cases $\mathrm{G}$ and $\mathrm{H}$. To explain this similarity let us focus, e.g., on the neutral VBF and HS processes (figure 19 and figure 21). ${ }^{7}$

In the neutral VBF process, out of all the involved diagrams (figure 19a,b,c) there is only one diagram, figure 19a, which depends on the Higgs-DM coupling. The cross-section of this diagram $\left(\sigma_{h}\right)$ for a given $m_{\mathrm{DM}}$ relative to the cross-section of all three diagrams involved $\left(\sigma_{\text {tot }}\right)$ is $\sigma_{h} / \sigma_{\text {tot }}=0.1445$ for case $\mathrm{G}$ and $\sigma_{h} / \sigma_{\text {tot }}=0.2416$ for case H. Recall that the main difference between cases $\mathrm{G}$ and $\mathrm{H}$ is that for a chosen $m_{\mathrm{DM}}$ the Higgs-DM

\footnotetext{
${ }^{7}$ The same argument applies to comparing the charged VBF and HS processes.
} 


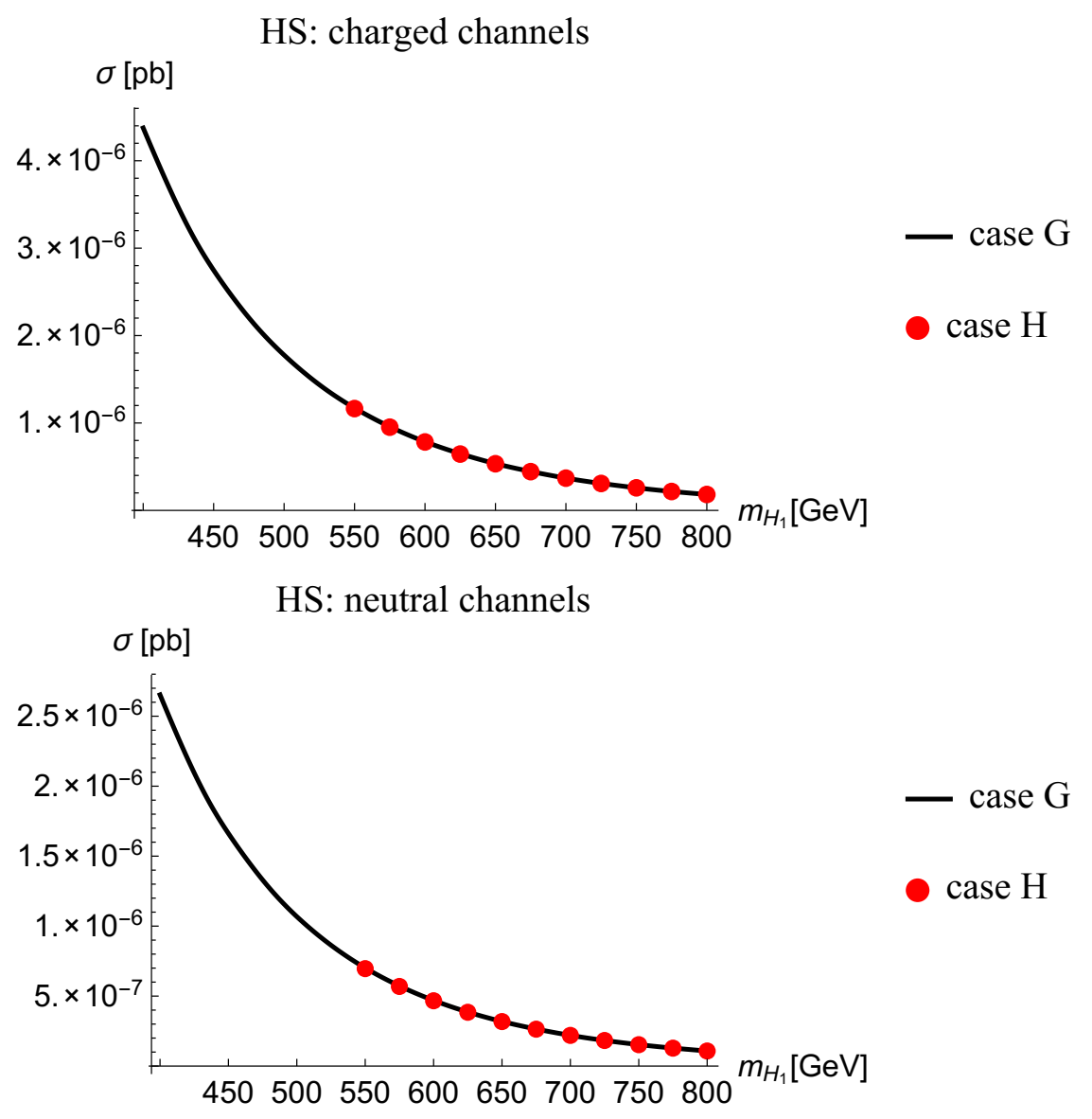

Figure 11. The cross-section in HS processes in terms of the DM mass in charged (top) and neutral (bottom) processes.

is larger in case $\mathrm{G}$ than in case $\mathrm{H}$. We conclude that the Higgs-mediated diagram plays a much more important role in case $\mathrm{H}$ than it does in case $\mathrm{G}$. As a result, even though the $g_{h H_{1} H_{1}}$ coupling is much smaller in case $\mathrm{H}$, the total cross-section does not fall far below the total cross-section in case G, which is depicted in figure 10 .

Now, let us consider the HS neutral processes (figure 21a,b,c). Again, only one diagram, figure 21a, depends on the Higgs-DM coupling. Repeating the procedure above, we calculate the relative cross-section of this one diagram $\left(\sigma_{h}\right)$ relative to the cross-section of all diagrams involved $\left(\sigma_{\text {tot }}\right)$. For a given $m_{\mathrm{DM}}$, we obtain $\sigma_{h} / \sigma_{\text {tot }}=0.9736$ in case $\mathrm{G}$ and $\sigma_{h} / \sigma_{\text {tot }}=0.9708$ in case $\mathrm{H}$. So, this diagram plays only a slight role in case $\mathrm{H}$ compared to case G. We therefore conclude that the difference in $g_{h H_{1} H_{1}}$ coupling does not play an important role in distinguishing case $\mathrm{G}$ and $\mathrm{H}$ in the $\mathrm{HS}$ processes. Thus, we do not expect to see a difference between cases $\mathrm{G}$ and $\mathrm{H}$, which is depicted in figure 11, with the important exception that case $\mathrm{G}$ allows for the heavy Higgs DM mass to be below $525 \mathrm{GeV}$, making its cross-section significantly larger. 


\section{Conclusion}

We have calculated the relic density for heavy Higgs DM in the $\mathrm{I}(2+1) \mathrm{HDM}$ and shown that the prospects for its discovery at both DD experiments and the LHC are significantly enhanced as compared to the I(1+1)HDM, where the heavy Higgs DM particle must have a mass above about $525 \mathrm{GeV}$ and is weakly coupled to the observed Higgs boson. Adding a second inert Higgs doublet helps to make the heavy Higgs DM region accessible to both DD and the LHC, by either increasing its couplings to the observed Higgs or lowering its mass to $360 \mathrm{GeV} \lesssim m_{\mathrm{DM}}$, or both. In particular we have presented LHC signatures of the $\mathrm{I}(2+1) \mathrm{HDM}$ in the monojet, VBF (dijet) and HS processes and shown that the prospects for heavy Higgs DM discovery are significantly brighter for all channels.

In DD experiments, although the standard values of annihilation cross-section for the heavy Higgs DM masses in the I $(2+1) \mathrm{HDM}$ are well below current experimental exclusion limits for DM decaying into pairs of gauge bosons or fermions, the prospects for a future DD discovery remain open due to the complementary nature of collider vs cosmological limits and the fact that the DD cross-sections are higher than in the I(1+1)HDM.

Turning to indirect detection signatures of the $\mathrm{I}(2+1) \mathrm{HDM}$, there is the possibility of internal bremsstrahlung in the processes of $H_{1} H_{1} \rightarrow W^{+} W^{-} \gamma$, generated through the exchange of any of the two charged scalars $H_{1,2}^{ \pm}$. It was shown that one can expect such signatures in the I(1+1)HDM [25], mediated by a charged scalar in the $t$-channel, which would correspond to scenario $\mathrm{H}$ considered in the $\mathrm{I}(2+1) \mathrm{HDM}$. In principle, the signal could even be stronger for scenario $\mathrm{G}$, as the scalar couplings are enhanced.

Finally we comment on the observed $h \rightarrow \gamma \gamma$ channel where, in the I(1+1)HDM, only in the heavy DM mass region are both proper DM relic density and (minimal) enhancement in the $h \rightarrow \gamma \gamma$ channel realised. By contrast, in the I(2+1)HDM there exist two charged

scalars, $H_{1}^{ \pm}$and $H_{2}^{ \pm}$, which contribute to the $h \rightarrow \gamma \gamma$ loop which may enhance the rate for a wide range of parameters.

In conclusion, adding a second inert Higgs doublet significantly improves the prospects for observability of heavy Higgs dark matter in future experiments both underground and at the CERN LHC.

\section{Acknowledgments}

SFK acknowledges partial support from the STFC Consolidated ST/J000396/ 1 and European Union FP7 ITN-INVISIBLES (Marie Curie Actions, PITN-GA-2011-289442). SM is financed in part through the NExT Institute and from the STFC Consolidated ST/ J000396/1. He also acknowledges the H2020-MSCA-RICE-2014 grant no. 645722 (NonMinimalHiggs). VK's research is financially supported by the Academy of Finland project "The Higgs Boson and the Cosmos" and project 267842. 


\section{A Feynman rules in the simplified I(2+1)HDM}

Here we present the Feynman rules of the model.

$$
\begin{array}{ll}
H_{2}^{+} H_{2}^{-} h, H_{1}^{+} H_{1}^{-} h & \lambda_{23} v \\
H_{1} H_{1} h, H_{2} H_{2} h & \left(\lambda_{23}+\lambda_{23}^{\prime}+2 \lambda_{2}\right) \frac{v}{2} \\
A_{1} A_{1} h, A_{2} A_{2} h & \left(\lambda_{23}+\lambda_{23}^{\prime}-2 \lambda_{2}\right) \frac{v}{2} \\
H_{2}^{+} H_{2}^{-} \gamma, H_{1}^{+} H_{1}^{-} \gamma & \frac{i}{2}\left(g \sin \theta_{W}+g^{\prime} \cos \theta_{W}\right)\left(K+K^{\prime}\right)^{\mu} \\
H_{2}^{+} H_{2}^{-} Z, H_{1}^{+} H_{1}^{-} Z & \frac{i}{2}\left(g \cos \theta_{W}-g^{\prime} \sin \theta_{W}\right)\left(K+K^{\prime}\right)^{\mu} \\
H_{1}^{ \pm} H_{1} W^{ \pm}, H_{2}^{ \pm} H_{2} W^{ \pm} & \frac{i g}{2} \cos \left(\theta_{h}-\theta_{c}\right)\left(K+K^{\prime}\right)^{\mu} \\
H_{2}^{ \pm} H_{1} W^{ \pm}, H_{1}^{ \pm} H_{2} W^{ \pm} & \frac{i g}{2} \sin \left(\theta_{h}-\theta_{c}\right)\left(K+K^{\prime}\right)^{\mu} \\
H_{1}^{ \pm} A_{1} W^{ \pm}, H_{2}^{ \pm} A_{2} W^{ \pm} & \frac{g}{2} \cos \left(\theta_{a}-\theta_{c}\right)\left(K+K^{\prime}\right)^{\mu} \\
H_{2}^{ \pm} A_{1} W^{ \pm}, H_{1}^{ \pm} A_{2} W^{ \pm} & \frac{g}{2} \sin \left(\theta_{a}-\theta_{c}\right)\left(K+K^{\prime}\right)^{\mu} \\
H_{1} A_{1}, H_{2} A_{2} Z & \frac{1}{2}\left(g \cos \theta_{W}+g^{\prime} \sin \theta_{W}\right) \cos \left(\theta_{h}-\theta_{a}\right)\left(K+K^{\prime}\right)^{\mu} \\
H_{2} A_{1}, H_{1} A_{2} Z & \frac{1}{2}\left(g \cos \theta_{W}+g^{\prime} \sin \theta_{W}\right) \sin \left(\theta_{h}-\theta_{a}\right)\left(K+K^{\prime}\right)^{\mu}
\end{array}
$$

where $K$ and $K^{\prime}$ are the momenta of the associated particles in the decay channel and $\theta_{W}$ is the Weak mixing angle. The Yukawa couplings in the model are identical to those of the SM.

\section{B Feynman diagrams for relic density calculations}

Here we present the DM (co)annihilation diagrams which play a role in our relic density studies.

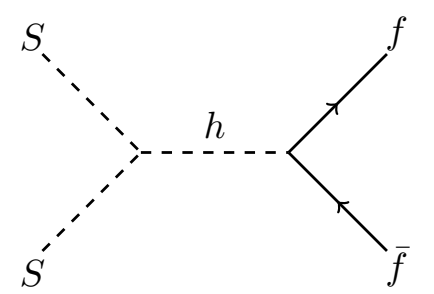

(a)

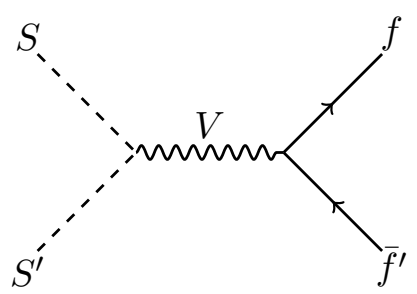

(b)

Figure 12. For light DM masses the most important channel for the annihilation of DM particles is the Higgs-mediated process (a). Coannhilation with other neutral scalars could have a significant effect on the relic density (b). 


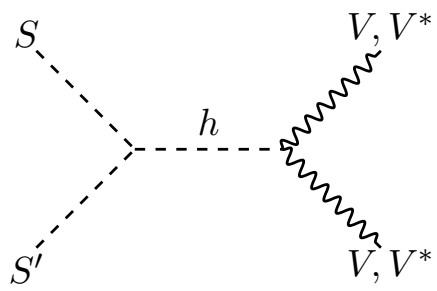

(a)

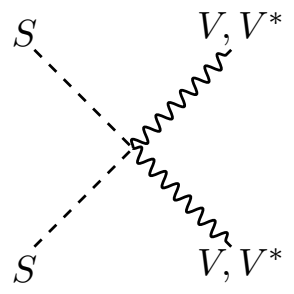

(b)

Figure 13. For heavy DM masses, the processes involving one or two virtual gauge bosons, also contribute to the total annihilation cross-section and affect the relic density.

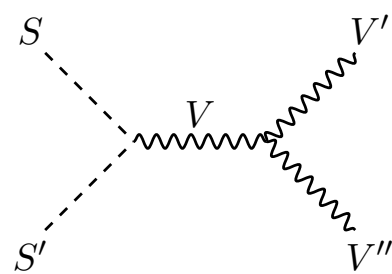

(a)

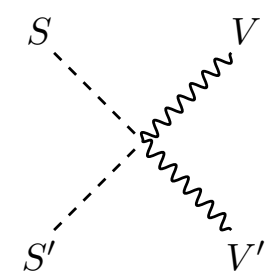

(b)

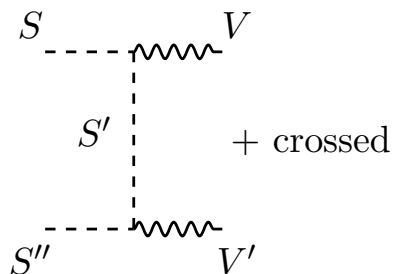

(c)

Figure 14. Heavy DM (co)annihilation diagrams with pure gauge boson final states.

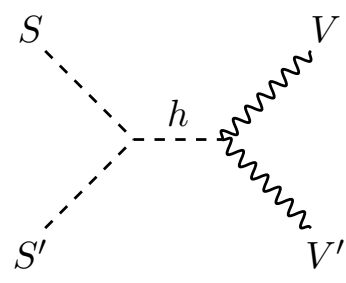

(a)

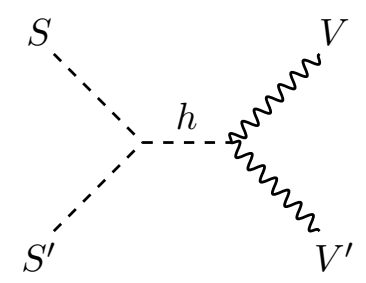

(b)

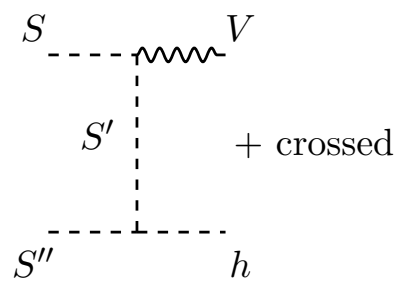

(c)

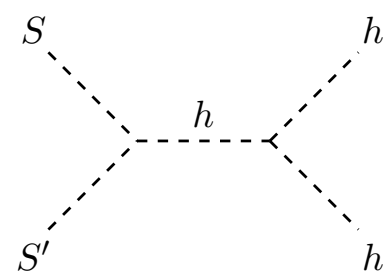

(d)

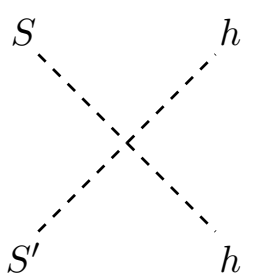

(e)

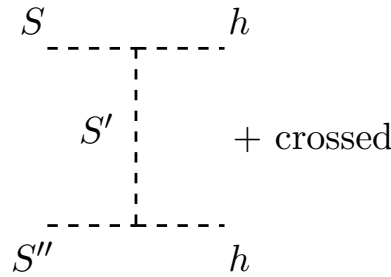

(f)

Figure 15. Heavy DM (co)annhilation channels involving the SM-like Higgs boson. 


\section{Feynman diagrams for the LHC analysis}

Here we present the DM (co)annihilation diagrams which play a role in our LHC studies.

\section{C.1 Diagrams with monojet final states}

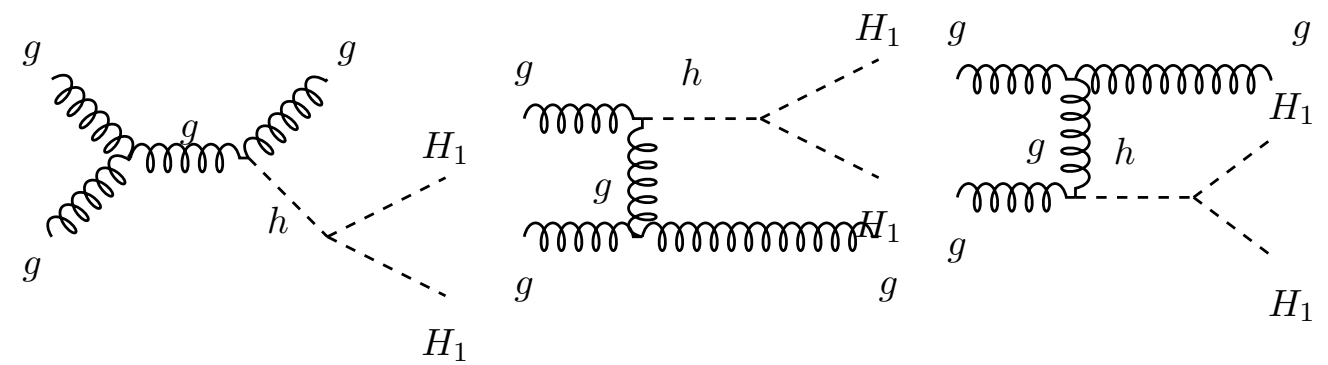

(a)

(b)

(c)

Figure 16. Relevant monojet diagrams with initial gluon states $\left(g g \rightarrow h g \rightarrow g H_{1} H_{1}\right)$ containing triple gluon vertex and an effective $g g h$ vertex.

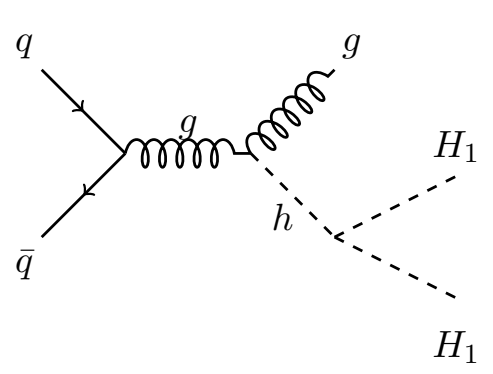

(a)

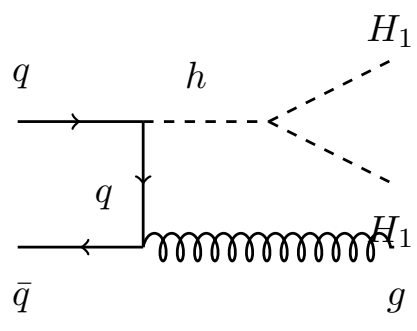

(b)

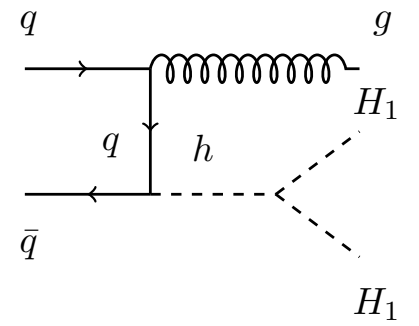

(c)

Figure 17. Relevant monojet diagrams with initial quark states $\left(q \bar{q} \rightarrow g H_{1} H_{1}+\right.$ diagrams with initial particles reversed) containing $g g h$ effective vertex, where $q=u, d, c, s, b$.

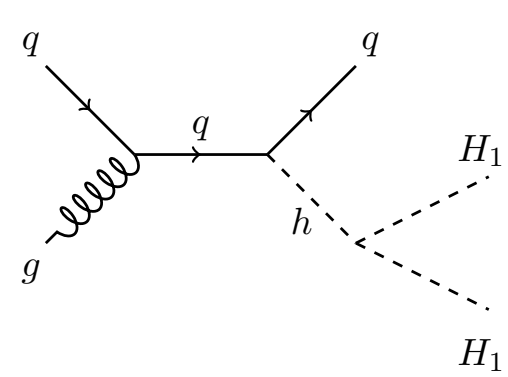

(a)

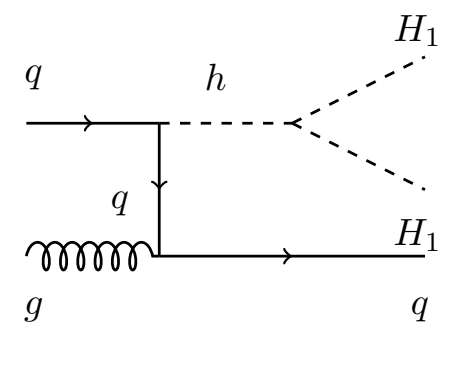

(b)

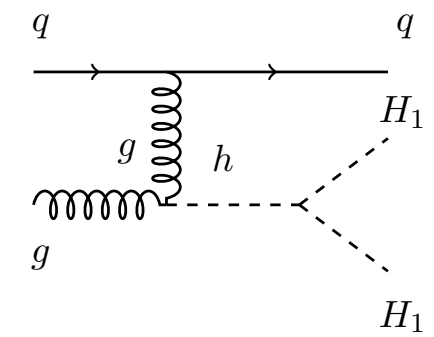

(c)

Figure 18. Relevant monojet diagrams with initial quark and gluon states $\left(q g \rightarrow q H_{1} H_{1}+\right.$ equivalent $\bar{q} g \rightarrow \bar{q} H_{1} H_{1}$ diagrams + diagrams with initial particles reversed) containing $g g h$ effective vertex, where $q=u, d, c, s, b$. 


\section{C.2 VBF diagrams with dijet final states}

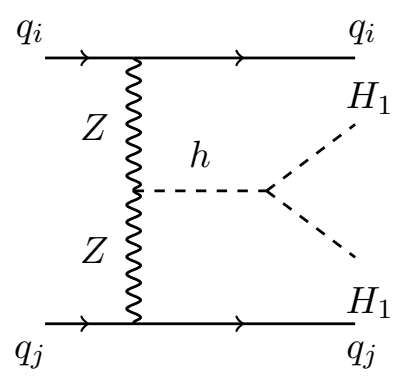

(a)

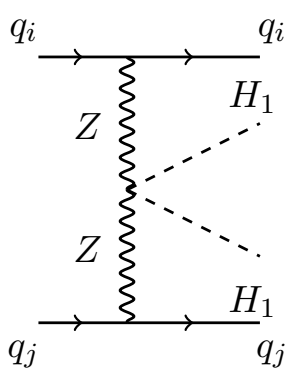

(b)

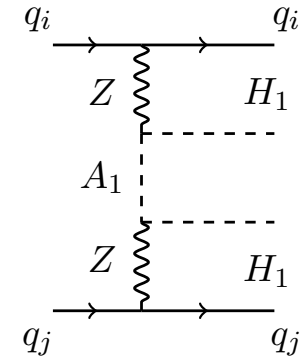

(c)

Figure 19. Relevant VBF diagrams with dijet final states $\left(q_{i} q_{j} \rightarrow H_{1} H_{1} q_{i} q_{j}\right)$ with only neutral intermediate gauge bosons, where $q=u, d$. Note that only one of the involved diagrams in this process depends on the value of the Higgs-DM coupling.

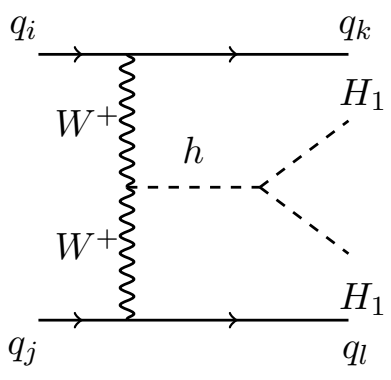

(a)

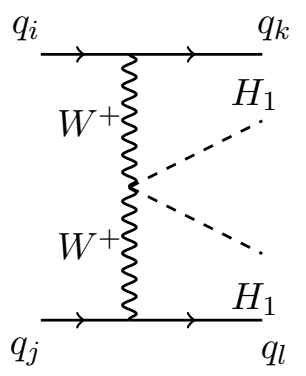

(b)

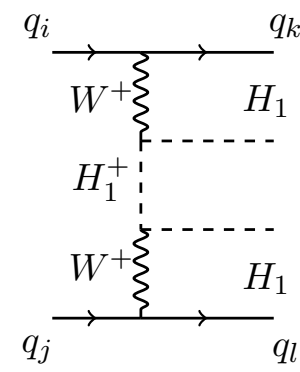

(c)

Figure 20. Relevant VBF diagrams with dijet final states $\left(q_{i} q_{j} \rightarrow H_{1} H_{1} q_{k} q_{l}\right)$ with only charged intermediate gauge bosons, where $q=u, d$. Note that only one of the involved diagrams in this process depends on the value of the Higgs-DM coupling.

\section{C.3 HS diagrams with (on-shell) gauge boson final states}

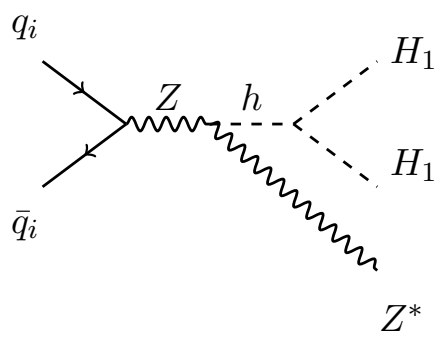

(a)

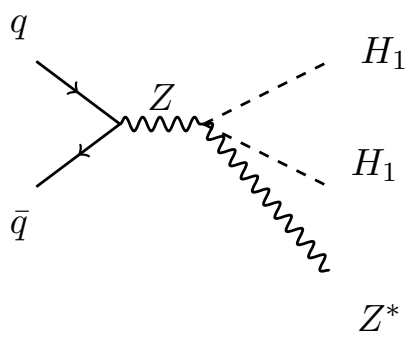

(b)

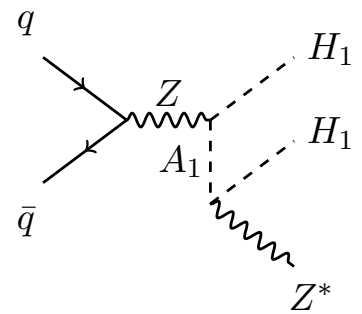

(c)

Figure 21. Relevant HS diagrams with (on-shell) neutral gauge boson final states $\left(q_{i} \bar{q}_{i} \rightarrow H_{1} H_{1} Z^{*}\right)$ and only neutral intermediate gauge bosons, where $q=u, d$. Note that only one of the involved diagrams in this process depends on the value of the Higgs-DM coupling. 


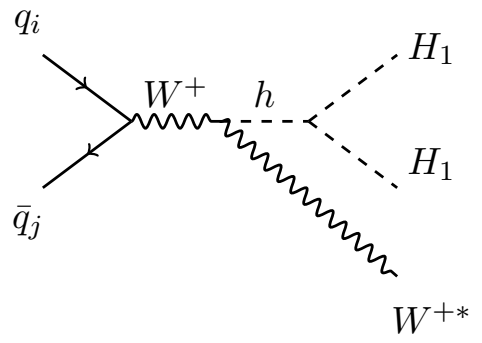

(a)

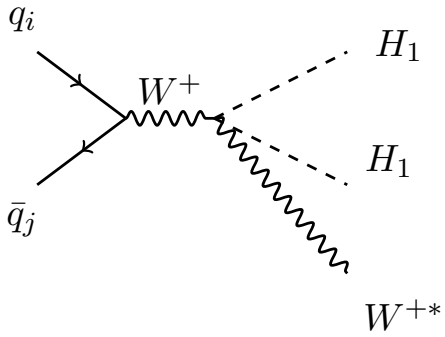

(b)

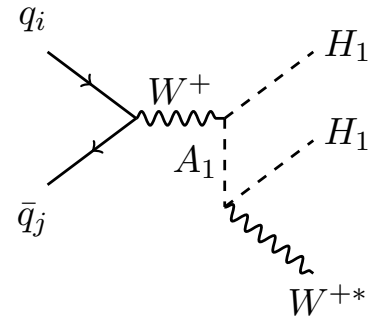

(c)

Figure 22. Relevant HS diagrams with (on-shell) charged gauge boson final states $\left(q_{i} \overline{q_{j}} \rightarrow\right.$ $H_{1} H_{1} W^{*+}$ ) and only charged intermediate gauge bosons, where $q=u, d$. Note that only one of the involved diagrams in this process depends on the value of the Higgs-DM coupling.

Open Access. This article is distributed under the terms of the Creative Commons Attribution License (CC-BY 4.0), which permits any use, distribution and reproduction in any medium, provided the original author(s) and source are credited.

\section{References}

[1] ATLAS collaboration, Observation of a new particle in the search for the Standard Model Higgs boson with the ATLAS detector at the LHC, Phys. Lett. B 716 (2013) 1 [arXiv:1207.7214] [INSPIRE].

[2] CMS collaboration, Observation of a new boson at a mass of $125 \mathrm{GeV}$ with the CMS experiment at the LHC, Phys. Lett. B 716 (2012) 30 [arXiv:1207.7235] [InSPIRE].

[3] Planck collaboration, P.A.R. Ade et al., Planck 2015 results. XIII. Cosmological parameters, arXiv:1502.01589 [INSPIRE].

[4] G. Jungman, M. Kamionkowski and K. Griest, Supersymmetric dark matter, Phys. Rept. 267 (1996) 195 [hep-ph/9506380] [INSPIRE].

[5] G. Bertone, D. Hooper and J. Silk, Particle dark matter: evidence, candidates and constraints, Phys. Rept. 405 (2005) 279 [hep-ph/0404175] [INSPIRE].

[6] L. Bergstrom, Nonbaryonic dark matter: observational evidence and detection methods, Rept. Prog. Phys. 63 (2000) 793 [hep-ph/0002126] [InSPIRE].

[7] N.G. Deshpande and E. Ma, Pattern of Symmetry Breaking with Two Higgs Doublets, Phys. Rev. D 18 (1978) 2574 [INSPIRE].

[8] S.F. King, Monojets From Z Decay Without Extra Neutrinos or Higgs, Phys. Rev. Lett. 54 (1985) 528 [INSPIRE].

[9] E. Ma, Verifiable radiative seesaw mechanism of neutrino mass and dark matter, Phys. Rev. D 73 (2006) 077301 [hep-ph/0601225] [INSPIRE].

[10] R. Barbieri, L.J. Hall and V.S. Rychkov, Improved naturalness with a heavy Higgs: an alternative road to LHC physics, Phys. Rev. D 74 (2006) 015007 [hep-ph/0603188] [INSPIRE]. 
[11] L. Lopez Honorez, E. Nezri, J.F. Oliver and M.H.G. Tytgat, The inert doublet model: an archetype for dark matter, JCAP 02 (2007) 028 [hep-ph/0612275] [INSPIRE].

[12] M. Krawczyk, D. Sokolowska, P. Swaczyna and B. Swiezewska, Constraining Inert Dark Matter by $R_{\gamma \gamma}$ and WMAP data, JHEP 09 (2013) 055 [arXiv: 1305.6266] [INSPIRE].

[13] A. Arhrib, Y.-L.S. Tsai, Q. Yuan and T.-C. Yuan, An updated analysis of inert Higgs doublet model in light of the recent results from LUX, PLANCK, AMS-02 and LHC, JCAP 06 (2014) 030 [arXiv:1310.0358] [INSPIRE].

[14] V. Keus, S.F. King, S. Moretti and D. Sokolowska, Dark matter with two inert doublets plus one Higgs doublet, JHEP 11 (2014) 016 [arXiv:1407.7859] [INSPIRE].

[15] I.P. Ivanov, V. Keus and E. Vdovin, Abelian symmetries in multi-Higgs-doublet models, J. Phys. A 45 (2012) 215201 [arXiv:1112.1660] [InSPIRE].

[16] E.M. Dolle and S. Su, The inert dark matter, Phys. Rev. D 80 (2009) 055012 [arXiv:0906.1609] [INSPIRE].

[17] S. Moretti and K. Yagyu, Constraints on Parameter Space from Perturbative Unitarity in Models with Three Scalar Doublets, Phys. Rev. D 91 (2015) 055022 [arXiv:1501.06544] [INSPIRE].

[18] LUX collaboration, D.S. Akerib et al., First results from the LUX dark matter experiment at the Sanford Underground Research Facility, Phys. Rev. Lett. 112 (2014) 091303 [arXiv: 1310.8214] [INSPIRE].

[19] XENON1T collaboration, E. Aprile, The XENON1T Dark Matter Search Experiment, Springer Proc. Phys. 148 (2013) 93 [arXiv:1206.6288] [INSPIRE].

[20] A.J. Anderson, J.M. Conrad, E. Figueroa-Feliciano, K. Scholberg and J. Spitz, Coherent Neutrino Scattering in Dark Matter Detectors, Phys. Rev. D 84 (2011) 013008 [arXiv: 1103.4894] [INSPIRE].

[21] L. Lopez Honorez and C.E. Yaguna, A new viable region of the inert doublet model, JCAP 01 (2011) 002 [arXiv: 1011.1411] [INSPIRE].

[22] V. Keus, S.F. King and S. Moretti, Phenomenology of the inert $(2+1)$ and (4+2) Higgs doublet models, Phys. Rev. D 90 (2014) 075015 [arXiv:1408.0796] [InSPIRE].

[23] A. Semenov, LanHEP - a package for automatic generation of Feynman rules from the Lagrangian. Updated version 3.2, arXiv:1412.5016 [INSPIRE].

[24] A. Belyaev, N.D. Christensen and A. Pukhov, CalcHEP 3.4 for collider physics within and beyond the Standard Model, Comput. Phys. Commun. 184 (2013) 1729 [arXiv:1207.6082] [INSPIRE].

[25] C. Garcia-Cely and A. Ibarra, Novel Gamma-ray Spectral Features in the Inert Doublet Model, JCAP 09 (2013) 025 [arXiv: 1306.4681] [INSPIRE]. 